\title{
Neurabin Scaffolding of Adenosine Receptor and RGS4 Regulates Anti-Seizure Effect of Endogenous Adenosine
}

\author{
Yunjia Chen, ${ }^{1}$ Yin Liu, ${ }^{1}$ Christopher Cottingham, ${ }^{1}$ Lori McMahon, ${ }^{1}$ Kai Jiao, ${ }^{2}$ Paul Greengard, ${ }^{3}$ and Qin Wang ${ }^{1}$ \\ Departments of ${ }^{1}$ Physiology and Biophysics, ${ }^{2}$ Genetics, University of Alabama at Birmingham, Birmingham, Alabama 35294, and ${ }^{3}$ Laboratory of Molecular \\ and Cellular Neuroscience, Rockefeller University, New York, New York 10065
}

\begin{abstract}
Endogenous adenosine is an essential protective agent against neural damage by various insults to the brain. However, the therapeutic potential of adenosine receptor-directed ligands for neuroprotection is offset by side effects in peripheral tissues and organs. An increase in adenosine receptor responsiveness to endogenous adenosine would enhance neuroprotection while avoiding the confounding effects of exogenous ligands. Here we report novel regulation of adenosine-evoked responses by a neural tissue-specific protein, neurabin. Neurabin attenuated adenosine $A_{1}$ receptor (A1R) signaling by assembling a complex between the A1R and the regulator of G-protein signaling 4 (RGS4), a protein known to turn off G-protein signaling. Inactivation of the neurabin gene enhanced A1R signaling and promoted the protective effect of adenosine against excitotoxic seizure and neuronal death in mice. Furthermore, administration of a small molecule inhibitor of RGS4 significantly attenuated seizure severity in mice. Notably, the dose of kainate capable of inducing an $\sim 50 \%$ rate of death in wild-type (WT) mice did not affect neurabin-null mice or WT mice cotreated with an RGS4 inhibitor. The enhanced anti-seizure and neuroprotective effect achieved by disruption of the A1R/neurabin/RGS4 complex is elicited by the on-site and ondemand release of endogenous adenosine, and does not require administration of A1R ligands. These data identify neurabin-RGS4 as a novel tissue-selective regulatory mechanism for fine-tuning adenosine receptor function in the nervous system. Moreover, these findings implicate the A1R/neurabin/RGS4 complex as a valid therapeutic target for specifically manipulating the neuroprotective effects of endogenous adenosine.
\end{abstract}

\section{Introduction}

The severity of neural damage is a key factor in determining the mortality and morbidity under pathological conditions such as hypoxia, ischemia, and excitotoxin exposure. Extracellular adenosine released under these conditions acts as a potent protective agent to reduce neural damage and to confine the development and progression of accompanying seizures that result from hyperexcitability (Cunha, 2001; Dunwiddie and Masino, 2001; Stone, 2002; Fredholm et al., 2005). Among the four subtypes $\left(A_{1}, A_{2 A}, A_{2 B}\right.$, and $\left.A_{3}\right)$ of adenosine receptors, the $A_{1}$ subtype (A1R) is the primary mediator of adenosine-evoked anticonvulsant and neuroprotective effects (Cunha, 2001; Dunwiddie and

Received Aug. 9, 2011; revised Dec. 21, 2011; accepted Dec. $28,2011$.

Author contributions: Q.W. supervised the entire project. Y.C., Y.L., and C.C. performed the experiments. L.M., K.J., P.G., and Q.W. were involved in experimental design and data analysis. Y.C., C.C., L.M., K.J., P.G. and Q.W. prepared the manuscript.

This research is supported by The University of Alabama at Birmingham (UAB) Faculty Development Grant (Q.W.) and National Institutes of Health (NIH) Grants MH081917 (Q.W.) and DA10044 (P.G.).We are grateful to Dr. R. Colbran (Vanderbilt University, Nashville, TN) for neurabin constructs GST-Nrb146-453 and Myc-Nrb, and for neurabin antibody; Dr. J. Hablitz (University of Alabama at Birmingham) for suggestions on seizure studies; and The UAB Behavioral Neuroscience Core (supported by NIH Grants NS 047466 and NS 057098) for electroencephalography analysis. We also thank Drs. L. Limbird and R. Colbran (Vanderbilt University) for critical reading of the manuscript. The authors declare no competing financial interests.

Correspondence should be addressed to Dr. Qin Wang, 986 MCLM, 1918 University Boulevard, Birmingham, AL 35294. E-mail: qinwang@uab.edu.

Y. Liu's present address: Department of Radiology, The Third XiangYa Hospital of Central South University, Changsha 410078 Hunan, China.

DOI:10.1523/JNEUROSCI.4125-11.2011

Copyright $\odot 2012$ the authors $\quad 0270-6474 / 12 / 322683-13 \$ 15.00 / 0$
Masino, 2001; Stone, 2002; Fredholm et al., 2005; Sebastião and Ribeiro, 2009). However, targeting the A1R for treatment of neurological diseases has been extremely challenging due to its broad expression in multiple peripheral tissues (Stone, 2002). Thus, using A1R-directed ligands likely evokes simultaneous peripheral actions that result in severe side effects and thereby confound therapeutic outcomes.

The A1R belongs to the G-protein-coupled receptor (GPCR) superfamily. Cumulative evidence has demonstrated that the magnitude and duration of GPCR signaling is tightly regulated by non-G-protein GPCR-interacting partners (for review, see Lefkowitz, 2007; Allen et al., 2008; Ritter and Hall, 2009; Bockaert et al., 2010). The complexity of GPCR signaling regulation was first revealed by identification of a group of proteins called regulators of G-protein signaling (RGS), which functions as GTPaseactivating proteins to terminate G-protein signaling (Ross and Wilkie, 2000; Hollinger and Hepler, 2002; Sjögren and Neubig, 2010). Association of RGS proteins with GPCRs, either directly or indirectly through adaptor proteins, represents the primary mechanism that determines the selectivity and effectiveness of RGS-mediated attenuation of GPCR signaling (Neubig and Siderovski, 2002; Neitzel and Hepler, 2006; Xie and Palmer, 2007). RGS4 belongs to the R4 subfamily of RGS proteins, and is highly expressed in brain and heart (Bansal et al., 2007). To date, involvement of RGS4 in adenosine-evoked signaling responses and neuroprotection remains largely unexplored.

Neurabin is a multidomain scaffolding protein specifically expressed in neural tissues (Nakanishi et al., 1997; Burnett et al., 
1998). Like the A1R (Reppert et al., 1991), a high-level expression of neurabin has been found in cortex, hippocampus, and cerebellum (Nakanishi et al., 1997; Burnett et al., 1998). Distribution of neurabin in both dendrites and presynaptic terminals (Muly et al., 2004) positions this protein as a potential regulator for both presynaptic and postsynaptic activities of neurons. Whether neurabin can directly interact with GPCRs to regulate receptor functions has not been determined.

Given the importance of non-G-protein-interacting partners in regulation of GPCR activity, we propose that modulating interactions between the A1R and neural-specific accessory proteins would provide an effective way to increase A1R responsiveness to endogenous adenosine. Thus, enhanced neuroprotection through the A1R can be achieved while avoiding the confounding effect of exogenous A1R ligands. Here, we identify a direct interaction between the A1R and neurabin, which attenuates A1R-mediated responses both in vitro and in vivo by scaffolding interaction between the active A1R and RGS4. Significantly, endogenous adenosine-elicited neuroprotection against excitotoxic seizures through the $\mathrm{A} 1 \mathrm{R}$ is enhanced in mice with a neurabin gene deficiency or when RGS4 is blocked by a small molecule inhibitor.

\section{Materials and Methods}

Reagents. Rat anti-HA monoclonal antibody was purchased from Roche Applied Science. Mouse HA.11 monoclonal antibody was from Covance. Mouse anti-Myc monoclonal antibody was from Clontech. Goat anti-RGS4 polyclonal antibody was from Santa Cruz Biotechnology. Rabbit anti-RGS4 polyclonal antibody was purchased from Abcam. Rabbit anti-A1R polyclonal antibody was from Pierce. Rabbit anti-GPF polyclonal antibody was from Santa Cruz Biotechnology. TNT in vitro translation kit was from Promega. The Biotrak cAMP competitive enzymeimmunoassay (EIA) system and $\left[{ }^{35} \mathrm{~S}\right]$ methionine were purchased from GE Healthcare. CCG-4986 [methyl- $N$-[(4-chlorophenyl) sulfonyl]4-nitro-benzenesulfinimidoate) was from the ChemBridge screening collection. Fluoro-Jade B was purchased from Histo-Chem. Hematoxylin and eosin (HE) solution was from the Zymed Laboratories of Invitrogen and Surgipath, respectively. The DeadEnd Fluorometric TUNEL System was from Promega. Kainate was from Milestone PharmTech. All other chemicals were from Sigma-Aldrich or Fisher Scientific.

Animals. Neurabin ${ }^{-1-}\left(\mathrm{Nrb}^{-1-}\right)$ mice (Allen et al., 2006) were backcrossed for 10 generations to the $\mathrm{C} 57 \mathrm{BL} / 6$ background. $\mathrm{Nrb}^{+/-}$mice were then mated to generate wild-type (WT) and $\mathrm{Nrb}^{-1-}$ littermates for behavioral experiments. Twelve-week-old male mice were used for in vivo studies. All mice were maintained at the University of Alabama at Birmingham Animal Care Facility in accordance with procedures of the Animal Welfare Act and the 1989 Amendments to this Act.

Cells and transfection. CosM6 cells were cultured in DMEM (Invitrogen) supplemented with 10\% FBS (Atlanta Biologicals) and $100 \mathrm{U} / \mathrm{ml}$ penicillin and $10 \mu \mathrm{g} / \mathrm{ml}$ streptomycin. CHO-K1 cells were cultured in DMEM/F-12 (Invitrogen) with 10\% FBS, penicillin and streptomycin, and $2 \mathrm{~mm}$ glutamine. Cells were transfected with Lipofectamine 2000 (Invitrogen).

Plasmid construction. cDNA encoding neurabin amino acids 146-330 and 331-453 were amplified by PCR and cloned into GST fusion vector
pGEX4T (GE Healthcare) through EcoRI and XhoI sites to obtain pGEX4T-Nrb146-330 and pGEX4T-Nrb331-453. cDNA encoding A1R amino acids 202-326, 202-292, and 236-326 were amplified by PCR and cloned into a pCMVTNT vector (Promega) through XhoI and KpnI sites to obtain pCMVTNT-A1R202-326, pCMVTNT-A1R202-292, and pCMVTNT-A1R236-326.

In vitro GST pull-down assay. Preparation of GST fusion proteins, synthesis of $\left[{ }^{35} \mathrm{~S}\right]$-labeled in vitro translated probes, and pull-down assays were performed as described previously (Wang and Limbird, 2002).

Coimmunoisolation assay. CosM6 cells were cotransfected with cDNA encoding HA-A1R together with cDNA encoding Myc-Nrb, Myc-Sp, or RGS4, or both Myc-Nrb and RGS4. At 48 h post-transfection, cells were stimulated with $1 \mu \mathrm{M} R$-phenylisopropyladenosine (R-PIA) for $5 \mathrm{~min}$. Cells were lysed in immunoisolation buffer containing $20 \mathrm{~mm}$ HEPES, pH 7.4, 2 mм EDTA, 5 mm NaF, 0.5\% NP-40, 10\% glycerol, and protease inhibitors. Rat anti-HA antibody and goat anti-RGS4 antibody were used to immunoisolate HA-A1R and RGS4, respectively, following a procedure described previously (Xu et al., 2008). Immunoisolates were separated by SDS-PAGE and analyzed by Western blot.

For coimmunoisolation of endogenous neurabin and A1R from brain lysate, adult male mice aged 12 weeks were injected with saline or R-PIA $(1 \mathrm{mg} / \mathrm{kg}$ ) and killed $30 \mathrm{~min}$ postinjection. Whole brain was removed by dissection and homogenized on ice in lysis and immunoisolation buffer as described above. The detergent extract was then centrifuged at $100,000 \times g$ at $4^{\circ} \mathrm{C}$ for $30 \mathrm{~min}$, and the resulting supernatant was subjected to immunoprecipitation assays with a rabbit polyclonal antibody against either the A1R or green fluorescent protein (GFP) (as a negative control). 


\begin{tabular}{|c|c|c|c|c|c|c|}
\hline \multirow{2}{*}{$\begin{array}{l}\text { A } \\
\text { Neurabin }\end{array}$} & \multicolumn{2}{|r|}{146} & 454 & \multicolumn{3}{|r|}{1095} \\
\hline & Actin & 1 & |PP1 & PDZ & $\mid$ Coiled-Coill & | SAM $\| D$ \\
\hline & $40 \%$ & $23 \%$ & $63 \%$ & $81 \%$ & $74 \%$ & \\
\hline Spinophilin & $\begin{array}{l}1 \\
\text { Actin }\end{array}$ & 155 & $\begin{array}{l}445 \\
\text { PP1 }\end{array}$ & & | & \\
\hline
\end{tabular}

B

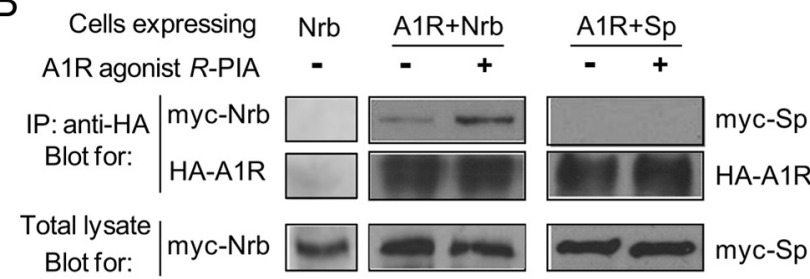

C
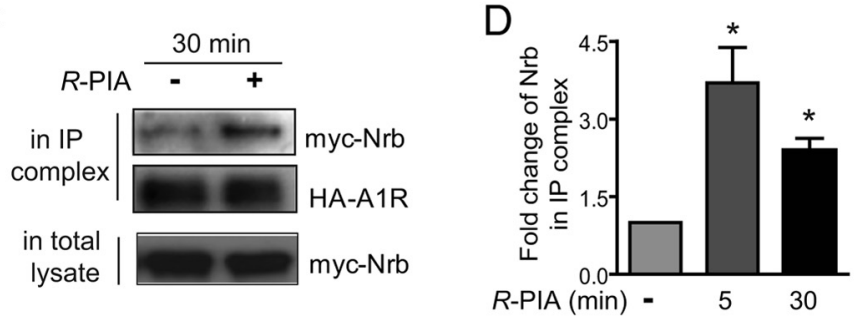

E

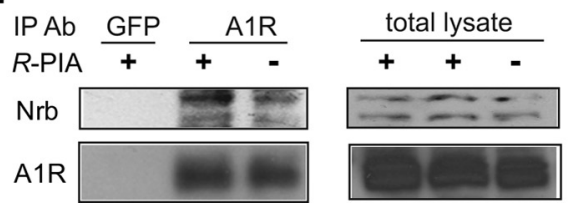

Figure 2. Agonist exposure specifically promotes interaction of the A1R with neurabin (Nrb) but not its homolog spinophilin in intact cells. $\boldsymbol{A}$, Sequence homology between domains in neurabin and spinophilin. $\boldsymbol{B}$, Interaction of the A1R with neurabin (left) or spinophilin (right) in cells treated with or without A1R agonist. Cells expressing neurabin alone or coexpressing HA-A1R with Myc-Nrb or Myc-Sp were stimulated with or without $1 \mu \mathrm{M}$ R-PIA for $5 \mathrm{~min}$, and cell lysates were subjected to immunoisolation assay using an antibody against HA. C, The A1R-neurabin interaction detected with prolonged R-PIA treatment for $30 \mathrm{~min}$. Cells coexpressing HA-A1R and Myc-Nrb were stimulated with $1 \mu \mathrm{m}$ R-PIA or vehicle for $30 \mathrm{~min}$, and cell lysates were subjected to immunoisolation assay. $\boldsymbol{D}$, Quantitation of A1R-neurabin interaction representing three to six independent coimmunoisolation experiments. Data are expressed as the fold change of neurabin in complex with the A1R over no stimulation control (defined as onefold). Values are given as the mean $\pm S E M ;{ }^{*} p<0.05$, R-PIA stimulated versus control. $\boldsymbol{E}$, Endogenous interaction between neurabin and A1R in mouse brain. Mice were given intraperitoneal injections of saline or R-PIA (1 mg/kg), with whole brains isolated and homogenized $30 \mathrm{~min}$ postinjection. Detergent-solubilized fractions were then prepared and subjected to coimmunoisolation using equal concentrations of an antibody against either GFP (negative control) or the A1R. Representative blots from three independent experiments are shown. A degradation product of the endogenous neurabin was also detected.

Antisense knockdown of RGS4 and RT-PCR. CHO-K1 cells were transfected with the RGS4 antisense oligo (5'-GAATCCAGGTTCACC-3') and scrambled oligo ( $5^{\prime}$-GATCGA ACT CGC TCA-3') (Witt-Enderby et al., 2004). Forty-eight hours post-transfection, total RNA was prepared using TRIZOL reagent (Invitrogen). RT-PCR was performed with the OneStep RT-PCR kit (Qiagen) following the manufacturer's instructions. PCR primers for application of RGS4 were 5'-GCTTTCAAAGCTTT CCTGAAGT-3' and 5'-TGTGAGAATTAGGCACACTG-3', the product of which covers partial sequences of exon 3 and exon 4 .

Intact cell surface ELISA. Cell-surface HA-A1R expression in transfected $\mathrm{CHO}-\mathrm{K} 1$ cells was examined by the cell-surface ELISA methods as described previously (Brady et al., 2003).

Radioligand binding assays. Crude membrane fraction of brains from WT and $\mathrm{Nrb}^{-1-}$ mice was prepared as described previously (Maemoto et al., 1997). In brief, brain homogenates in $0.32 \mathrm{M}$ sucrose were centrifuged at $1000 \times g$ for $10 \mathrm{~min}$, and the resulting supernatants were centrifuged again at $17,000 \times g$ for $20 \mathrm{~min}$. The pellet was then resuspended in a buffer containing 10 mM HEPES, 10 mM EDTA, $\mathrm{pH}$ 7.4, with protease inhibitors and aspirated/expelled through a 20 gauge needle 10 times. After centrifugation at $40,000 \times g$ for $15 \mathrm{~min}$, the membrane pellet was washed twice in the HE buffer (10 mM HEPES, 1 mM EDTA, pH 7.4, with protease inhibitors). Membrane pellet was then resuspended in the HE buffer and protein concentrations were determined with the Bio-Rad DC Protein Assay Kit.

Saturation binding was performed to assess the A1R receptor density in brains of WT and $\mathrm{Nrb}^{-1-}$ mice using a method described earlier (Scholl and Wells, 2000). Membrane proteins $(150 \mu \mathrm{g} /$ reaction $)$ were incubated with increasing concentrations of $\left[{ }^{3} \mathrm{H}\right]$ DPCPX $(8-$ cyclopentyl-1,3-dipropylxanthine) in a total volume of $250 \mu \mathrm{l}$. Before the binding assay, membrane preparations were incubated with adenosine deaminase (ADA; $5 \mathrm{U} / \mathrm{mg}$ protein) at $25^{\circ} \mathrm{C}$ for $60 \mathrm{~min}$ to remove endogenous adenosine.

Competition binding was performed as described previously (Scholl and Wells, 2000) to evaluate the intrinsic affinity of agonist (RPIA) and antagonist (theophylline) at the A1R in brains of WT and $\mathrm{Nrb}^{-1-}$ mice, and $0.5 \mathrm{nM}$ $\left[{ }^{3} \mathrm{H}\right] \mathrm{DPCPX}$ was used as the radioligand. Assays were performed in the presence of $100 \mu \mathrm{M}$ $5^{\prime}$-guanylimidodiphosphate $(\mathrm{Gpp}(\mathrm{NH}) \mathrm{p})$ to eliminate effects of G-protein regulation of the receptor affinity for agonist. All binding data were analyzed using Prism software (GraphPad Software).

Primary culture of cortical neurons. Primary culture of cortical neurons from newborn mouse pups was performed following a protocol described earlier (Cottingham et al., 2011). In brief, newborn mouse cortices were dissected out, minced, and digested with papain for $15 \mathrm{~min}$ at $37^{\circ} \mathrm{C}$. Neurons were dissociated by trituration. Dissociated cortical neurons were plated in Neurobasal-A medium supplemented with 5\% FBS, 2\% B27, 2\% glutamax, $0.2 \%$ gentamycin at $5 \times 10^{4}$ cells/well in 24 well plates. From the second day, cells were fed with Neurobasal-A medium supplemented with $2 \%$ B $27,2 \%$ glutamax, $0.2 \%$ gentamycin, which were changed every $4-6 \mathrm{~d}$.

Measurement of cAMP levels. CHO-K1 cells were cotransfected with cDNA encoding HAA1R together with or without cDNA encoding Myc-Nrb or Myc-Sp. For RGS4 knock-down experiments, cells were also cotransfected with antisense or scrambled oligos. Cells were then seeded into 96-well plates $\left(4 \times 10^{4}\right.$ cells/well). Forty-eight hours post-transfection, cells were stimulated with $10 \mu \mathrm{M}$ forskolin with or without $1 \mu \mathrm{M}$ R-PIA for $20 \mathrm{~min}$ at $37^{\circ} \mathrm{C}$. cAMP levels in cells were measured using the GE Healthcare cAMP Biotrak EIA system following the manufacturer's instructions. To examine the effect of RGS4 inhibition on cAMP production, cells were pretreated with $30 \mu \mathrm{M}$ CCG4986 for $10 \mathrm{~min}$, and then stimulated with forskolin and R-PIA in the presence of CCG-4986.

cAMP levels were also measured in cortical neurons cultured for 9-11 $\mathrm{d}$ in vitro following a method described previously (Murphy et al., 1991) with slight modifications. Before agonist stimulation, neurons were incubated with ADA $(2 \mathrm{U} / \mathrm{ml})$ for $1 \mathrm{~h}$ to remove adenosine in medium. Neurons were then pretreated with $1 \mu \mathrm{M}$ R-PIA or vehicle for $10 \mathrm{~min}$, followed by cotreatment with $10 \mu \mathrm{M}$ forskolin (to activate adenylate cyclase) and $25 \mu \mathrm{M}$ Ro-20-1724 (to inhibit cAMP-specific phosphodiesterase) in the presence or absence of $1 \mu \mathrm{M}$ R-PIA for another $10 \mathrm{~min}$. cAMP levels were measured using the EIA system as described above. To examine the effect of RGS4 inhibition, $30 \mu \mathrm{M}$ CCG-4986 was added to cells together with forskolin and Ro-20-1724 in the presence or absence of R-PIA.

Immunofluorescence staining of cultured neurons. Cortical neurons cultured for 11-13 d in vitro were used for immunofluorescence studies. 
Before treatment, cells were washed in Neurobasal-A medium containing $0.5 \mathrm{U} / \mathrm{ml} \mathrm{ADA}$ for $30 \mathrm{~min}$. Cells then were treated with $1 \mu \mathrm{M}$ R-PIA or solvent for $5 \mathrm{~min}$, and fixed with 3\% paraformaldehyde and 3\% sucrose following treatment. Immunofluorescence staining was performed as described previously (Richman et al., 2001) using a Myc antibody or RGS4 antibody. Fluorescent images were taken under a confocal laser scanning microscope, and analyzed with MetaMorph software.

Measurement of sedative response. Twelve-week-old WT and $\mathrm{Nrb}^{-1-}$ mice were injected intraperitoneally with increasing doses of R-PIA or UK14,304. Sedative response was assessed by rotarod latency as described previously (Wang et al., 2004).

Induction and evaluation of seizure. Twelve-week-old WT and $\mathrm{Nrb}^{-1-}$ male mice were given one of the following treatments by intraperitoneal injection: (1) kainate at 20 or $25 \mathrm{mg} / \mathrm{kg}$ (dissolved in saline), (2) kainate ( $25 \mathrm{mg} / \mathrm{kg}$ in saline) together with DPCPX (0.5 mg/kg in saline), (3) with DMSO, (4) with CCG-4986 (20 mg/kg in DMSO), (5) with both DMSO and DPCPX $(0.5 \mathrm{mg} / \mathrm{kg}$ in saline), and (6) with both CCG-4986 (20 $\mathrm{mg} / \mathrm{kg}$ in DMSO $)$ and DPCPX (0.5 mg/kg in saline). Mice were observed for $2 \mathrm{~h}$ after kainate injection by trained observers blind to genotype or treatment. The severity of seizure behaviors was scored $0-7$ with 0 representing normal behavior and 7 representing death according to reference descriptions (Schauwecker and Steward, 1997; Yang et al., 1997). Seizure scores were recorded every $20 \mathrm{~min}$ for $2 \mathrm{~h}$.

Assessment of cell death in hippocampal slices. Seven days after initial kainate exposure, mice were deeply anesthetized with isoflurane and perfused with $4 \%$ paraformaldehyde in phosphate-buffered solution, $\mathrm{pH}$ 7.4. Hippocampal slices $(7 \mu \mathrm{m})$ were stained with HE. Neuronal death was detected by Fluoro-Jade B staining and by the DeadEnd Fluorometric TUNEL System (Promega) following the manufacturer's instructions.

Electroencephalography recordings. Fourteen weeks after kainate injection, mice were anesthetized with intraperitoneal injection of a ketamine/xylazine mixture. Electrodes were placed onto the dura mater bilaterally. After an overnight recovery, electroencephalography (EEG) activity of freely moving mice was monitored and recorded using a DSI NeuroScore CNS Analysis Software (Data Sciences International) for $24 \mathrm{~h}$.

Statistical analysis. All quantitative data were analyzed using Prism software (GraphPad Software). Seizure scores were analyzed by two-way ANOVA with Bonferroni's post-tests to compare replicate means. Genotype or treatment was considered as a between-subject factor and time was considered as a within-subject factor. Other data were analyzed with Student's $t$ tests (two-tail, unpaired). $p<0.05$ was considered statistically significant.

\section{Results}

Neurabin interacts with the adenosine A1R in an agonist-regulated manner

In our effort to search for A1R interacting proteins, we identified a direct interaction between the A1R and neurabin using a GST pull-down assay (Fig. 1A). The neurabin sequences that interacted with the A1R were mapped to amino acids 331-453 (Fig. $1 B$ ), within the region least conserved between neurabin and its close homolog, spinophilin (Fig. $2 \mathrm{~A}$ ). While both the third intracellular (3i) loop and C-terminal tail of A1R appeared to interact with neurabin, the $3 \mathrm{i}$ loop was required for the maximal level of interaction (Fig. 1C). Interaction between neurabin and the A1R in intact cells was markedly enhanced with stimulation by the A1R agonist R-PIA for 5 (Fig. 2 B, left) and $30 \mathrm{~min}$ (Fig. 2C), indicating that the A1R-neurabin interaction is regulated by receptor activation. Thus, an approximately threefold increase in the amount of neurabin coimmunoisolated with HA-tagged A1R was measured in cells stimulated with R-PIA compared with cells receiving no stimulation (Fig. $2 \mathrm{D}$ ). Moreover, systemic injection of R-PIA enhanced the amount of endogenous neurabin coimmunoisolated with the A1R in brain (Fig. 2E). Consistent with the fact that neurabin sequences interacting with the $A 1 R$ are distinct from its homolog, spinophilin, an interaction between
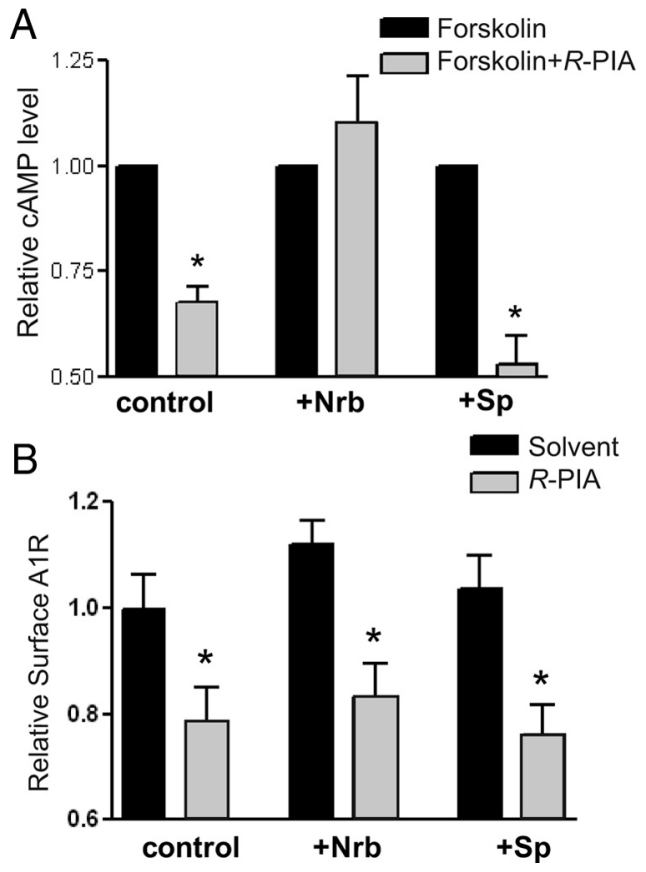

Figure 3. Neurabin attenuates A1R-mediated G-protein signaling in cells. $\boldsymbol{A}$, Neurabin expression attenuates A1R-mediated inhibition of cAMP production. Cells expressing HA-A1R alone or in combination with either neurabin (Nrb) or spinophilin (Sp) were treated with $10 \mu \mathrm{m}$ forskolin alone or forskolin plus $1 \mu \mathrm{m}$ R-PIA. Surface receptor density in each experimental group was comparable, as verified by intact cell ELISA using an anti-HA antibody. $n=11$ for cells expressing the A1R alone; $n=8$ for cells expressing the A1R and neurabin; $n=5$ for cells expressing the A1R and spinophilin. Data are expressed as the fold change in CAMP production over forskolin alone control (defined as onefold). ${ }^{*} p<0.05$, R-PIA stimulated versus control. $\boldsymbol{B}$, R-PIA induces similar levels of receptor internalization from the cell surface in cells either with or without exogenous expression of neurabin or spinophilin. Surface receptor density in cells described in $\boldsymbol{A}$ was examined by intact cell ELISA using an anti-HA antibody. ${ }^{*} p<0.05$, when comparing cell-surface A1R in R-PIA-treated cells versus nontreated controls.

the A1R and spinophilin was not detected in cells coexpressing these two proteins (Fig. $2 B$, right).

\section{Neurabin attenuates A1R-mediated responses in vitro and in vivo}

To determine the effects of the adenosine-evoked A1R-neurabin interaction on A1R signaling, we measured A1R-mediated inhibition of cAMP production stimulated by R-PIA. In CHO cells expressing the A1R, R-PIA stimulation caused inhibition of forskolin-induced cAMP production (Fig. $3 A$ ). When exogenous neurabin was expressed in $\mathrm{CHO}$ cells (which do not express endogenous neurabin), A1R-mediated inhibition of cAMP production was abolished (Fig. $3 A$ ), while cell surface expression of the A1R was not changed (Fig. $3 B$ ). These data suggest that neurabin interaction with the A1R leads to suppression of receptor signaling. Consistent with our inability to detect A1R-spinophilin interactions, overexpression of spinophilin had no effect on A1R-mediated inhibition of cAMP production (Fig. 3A).

Since the A1R-neurabin interaction attenuates A1R signaling, A1R-mediated in vivo responses should be enhanced in the absence of neurabin expression. To test this prediction, we assessed the ability of the A1R agonist R-PIA to evoke sedation mediated by the central A1R in WT versus neurabin-deficient $\left(\mathrm{Nrb}^{-/-}\right)$ mice (Allen et al., 2006). $\mathrm{Nrb}^{-1-}$ mice were more sensitive to R-PIA-evoked sedation, as manifest by a leftward shift of the dose-response curve (Fig. 4A). These data suggest that when neurabin is endogenously expressed, it negatively regulates A1R- 
A
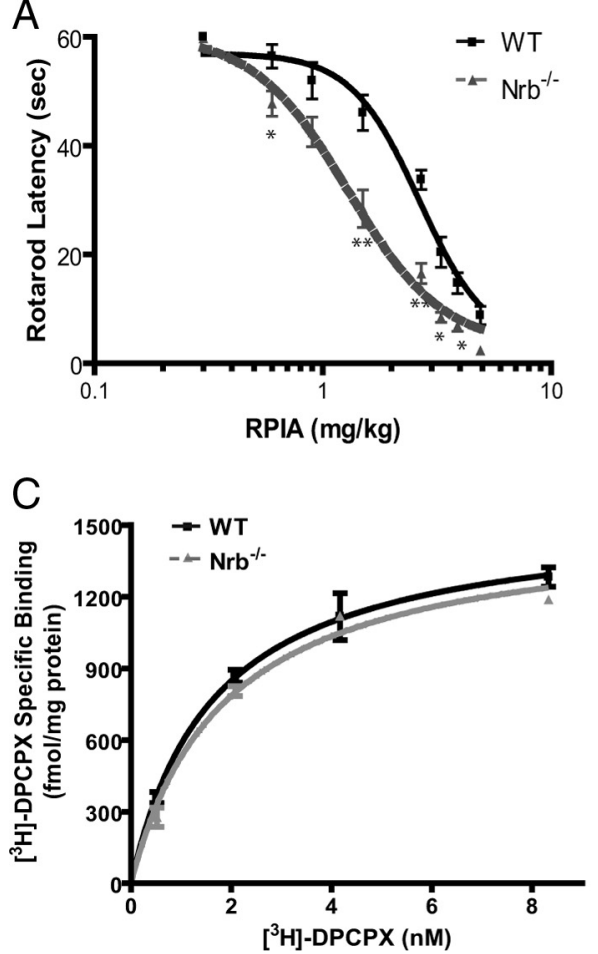

$\mathrm{B}$

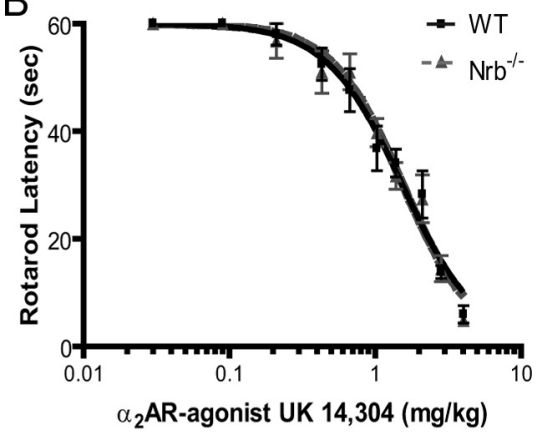

$\mathrm{D}$

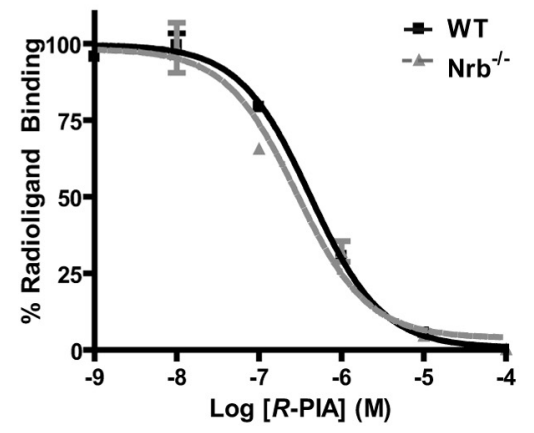

Figure 4. Neurabin attenuates A1R-mediated responses in vivo. $A$, Neurabin-deficient mice are more sensitive to R-PIA-elicited sedation, as assessed by rotarod latency. The $\mathrm{EC}_{50}$ values for sedation in $\mathrm{Nrb}^{-1-}(n=8)$ and corresponding WT littermates $(n=$ 5) are 1.29 and $2.64 \mathrm{mg} / \mathrm{kg}$, respectively. ${ }^{*} p<0.05 ;{ }^{* *} p<0.01 . B$, The $\alpha_{2}$ AR-agonist, UK14,304, induces comparable sedation responses in both WT and Nrb ${ }^{-1-}$ mice as assessed by rotarod latency. $n=5$ for both WT and Nrb ${ }^{-1-}$ mice. Error bars indicate mean \pm SEM. C, The density of A1Rs is indistinguishable in brain membrane preparations obtained from WT and Nrb ${ }^{-1-}$ mice as measured by saturation binding assays. Values represent mean $\pm \mathrm{SEM} ; n=3$ for each genotype. The $B_{\max }$ values predicted by nonlinear regression fit for the A1R in WT and $\mathrm{Nrb}^{-1-}$ brain homogenates are $1547 \pm 90$ and $1515 \pm 76 \mathrm{fmol} / \mathrm{mg}$ protein, respectively. $\boldsymbol{D}$, The intrinsic affinity of the A1R for R-PIA in brains of WT mice is similar to that in brains of $\mathrm{Nrb}^{-1-}$ mice. Competition binding assays were performed in the presence of $100 \mu \mathrm{m} \mathrm{Gpp}(\mathrm{NH}) \mathrm{p}$. Binding of the $\left[{ }^{3} \mathrm{H}\right] \mathrm{DPCPX}$ radioligand is given as a percentage of binding without competitors. Values represent mean $\pm \mathrm{SEM} ; n=3$ for each genotype. The $\mathrm{IC}_{50}$ values predicted for R-PIA in competition for radioligand binding in WT and $\mathrm{Nrb}^{-1-}$ brain homogenates are $0.41 \pm 0.11$ and $0.29 \pm 0.12 \mu \mathrm{M}$, respectively.

mediated responses in the brain, and this suppression is relieved in $\mathrm{Nrb}^{-1-}$ mice. We next measured the sedation response in these mutant mice evoked by the central $\alpha_{2}$ adrenergic receptor (AR), which does not interact with neurabin (Richman et al., 2001). As expected, the dose-response curve for sedation evoked by the $\alpha_{2} \mathrm{AR}$ agonist, UK14,304, was indistinguishable in WT and $\mathrm{Nrb}^{-1-}$ mice (Fig. $4 B$ ), suggesting that perturbation of A1R responses in $\mathrm{Nrb}^{-1-}$ mice is not generalized or nonspecific in nature.

The observed changes in sensitivity to A1R-evoked sedation in $\mathrm{Nrb}^{-1-}$ mice may result from alterations in A1R expression density and/or intrinsic affinity of the receptor for agonists caused by loss of neurabin in these mice. However, saturation binding analysis revealed that A1R density in the brains of WT and $\mathrm{Nrb}^{-1-}$ mice was indistinguishable, as was the $K_{\mathrm{d}}$ value for binding of the radiolabeled A1R antagonist, [ $\left.{ }^{3} \mathrm{H}\right] \mathrm{DPCPX}$ (Fig. 4C). Additionally, the intrinsic affinity of the A1R for its agonist R-PIA was nearly identical in brain membrane preparations from WT and $\mathrm{Nrb}^{-1-}$, as indicated by the $\mathrm{IC}_{50}$ values for competition of R-PIA with $\left[{ }^{3} \mathrm{H}\right]$ DPCPX (Fig. 4D).Together, our findings indicate that interactions between neurabin and the A1R lead to attenuation of A1R function in vivo without affecting A1R receptor density or its intrinsic affinity for agonists.
Functional involvement of RGS4 in neurabin-mediated inhibition of A1R signaling

Multiple molecular mechanisms could account for the ability of neurabin to suppress A1R cellular signaling through interactions with the receptor. For example, since GPCR signaling is attenuated by receptor phosphorylation and internalization (for review, see Ferguson, 2001; Reiter and Lefkowitz, 2006; Hanyaloglu and von Zastrow, 2008), neurabin interactions could enhance these processes. However, we failed to detect significant A1R phosphorylation in cells stimulated with R-PIA, even though significant agonist-stimulated $\alpha_{2 \mathrm{~A}} \mathrm{AR}$ phosphorylation was detectable under the same experimental conditions (data not shown). In addition, we did not find that overexpression of neurabin altered the extent of surface receptor internalization following R-PIA stimulation (Fig. 3B). These data suggest that neurabin attenuates A1R signaling via a mechanism that is independent of both receptor phosphorylation and internalization.

Mammalian RGS4 has been shown to accelerate GTPase activity of the $G_{i}$ subfamily of G-proteins, thus attenuating $\mathrm{G}_{\mathrm{i}^{-}}$ coupled signal transduction (Berman et al., 1996; Huang et al., 1997). We therefore tested the involvement of RGS4 in neurabin-mediated attenuation of A1R signaling by examining A1R-mediated inhibition of cAMP production in cells with altered RGS4 activity or blocked RGS4 expression. As shown above (Fig. 3A), R-PIA stimulation led to inhibition of cAMP production, and heterologous expression of neurabin diminished this A1R response (Fig. 5A). However, when neurabin-expressing cells were treated with an RGS4 inhibitor, CCG-4986 (Roman et al., 2007, 2010), neurabindependent loss of A1R-mediated inhibition of cAMP production was blocked (Fig. 5A). Suppression of RGS4 expression by antisense oligonucleotides (Fig. $5 B$ ) or small interfering RNA (Fig. $5 C)$ also diminished neurabin-mediated attenuation of A1R signaling. These data suggest that RGS4 is functionally involved in neurabin-mediated attenuation of A1R signaling in cells. Intriguingly, in cells without exogenous neurabin expression, the effect of RGS4 inhibition on cAMP production is negligible (Fig. 5A), indicating that neurabin is an essential component for RGS4induced attenuation of A1R signaling.

We next sought to verify that this observed regulation of A1R signaling by neurabin and RGS4 occurs in native neurons. In WT neurons, activation of endogenously expressed A1Rs by R-PIA resulted in $\sim 30 \%$ inhibition of cAMP production, whereas the same concentration of R-PIA led to a nearly $50 \%$ inhibition of cAMP production in neurons derived from $\mathrm{Nrb}^{-1-}$ mice (Fig. 5D). Blockade of RGS4 by CCG-4986 also significantly enhanced R-PIA-mediated inhibition of cAMP production in WT neurons (Fig. 5D). These data strongly support the role of neurabin and RGS4 in attenuating A1R signaling in native settings. Further- 
more, inhibition of RGS4 by CCG-4986 had no effect on R-PIA-mediated inhibition of cAMP production in neurons derived from $\mathrm{Nrb}^{-1-}$ mice (Fig. 5D), confirming that neurabin expression is required for RGS4 to induce attenuation of A1R signaling in the endogenous system.

\section{Neurabin scaffolds complex formation between the A1R and RGS4 and promotes membrane translocation of RGS4}

Though RGS proteins can interact directly with GPCRs (Neitzel and Hepler, 2006) to confer their selective regulation of GPCR signaling, we failed to detect direct interactions between the A1R and RGS4 in cells expressing these two proteins (Fig. 6A, left). On the other hand, in cells coexpressing neurabin with the $\mathrm{A} 1 \mathrm{R}$ and RGS4, both neurabin and RGS4 were coimmunoisolated with the A1R following R-PIA stimulation (Fig. 6A, right). Reciprocal immunoisolations using an antibody against RGS4 also pulled down the A1R and neurabin in an agonistdependent manner (Fig. 6B). These data are consistent with the interpretation that neurabin serves as a scaffold bridging functional interactions between the A1R and RGS4 in cells, and that agonist stimulation promotes A1R/neurabin/RGS4 complex formation. This notion also explains our findings that the RGS inhibitor, CCG-4986, does not alter R-PIA-elicited inhibition of cAMP production in cells unless neurabin is also expressed (Fig. 5A). In addition, R-PIA stimulation did not enhance the interaction of neurabin with RGS2 (Fig. 6C), another member of the R4 family of RGS proteins, demonstrating the selectivity of the neurabin-facilitated A1R-RGS interactions for RGS4.Together, our data suggest that the A1R, neurabin, and RGS4 form a complex following agonist stimulation to attenuate A1R signaling.

Plasma membrane localization of RGS4 is critical for its ability to inhibit G-protein signaling (Srinivasa et al., 1998). While RGS4 is largely localized in the cytoplasm of neuronal cells (Chatterjee and Fisher, 2000), neurabin is mainly localized to the plasma membrane via its actin-binding domain (Oliver et al., 2002). We postulated that neurabin recruits RGS4 to the plasma membrane to turn off G-protein signaling initiated by the A1R. To test this possibility, we examined RGS4 localization in primary cortical neurons cultured from WT or $\mathrm{Nrb}^{-1-}$ mouse brains. In WT neurons, RGS4, both when exogenously transfected (Fig. 7A) and when endogenously expressed (Fig. 7B), was mainly detected in cytoplasm of the majority of cells, and R-PIA stimulation caused translocation of RGS4 to the plasma membrane. In contrast, in $\mathrm{Nrb}^{-1-}$ neurons, R-PIA stimulation failed to induce efficient RGS4 translocation (Fig. $7 A, B$ ). These data suggest that neurabin serves as a scaffold to recruit RGS4 to the plasma membrane following A1R activation. forskolin.
$B$

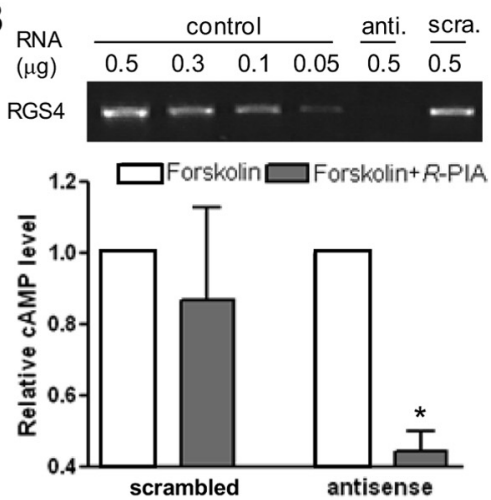

$\mathrm{D}$

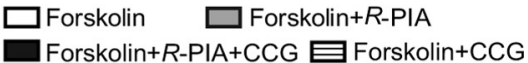

$\square$ Forskolin+R-PIA+CCG $\equiv$ Forskolin+CCG

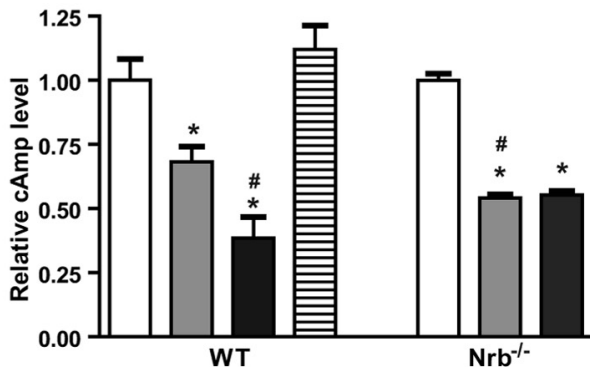

Figure 5. Involvement of RGS4 in neurabin-mediated attenuation of A1R signaling. A, RGS4 inhibitor CCG-4986 reverses treated with forskolin alone, forskolin plus R-PIA, or forskolin plus R-PIA and CCG-4986 (30 $\mu \mathrm{m})$. Data are from four independent ith cells expressing HA-A1R alone and from 10 independent experiments with cells expressing HA-A1R with neura${ }^{*} p<0.05$, indicated treatment versus forskolin alone. $\boldsymbol{B}$, Knockdown of RGS4 expression by antisense oligo diminishe

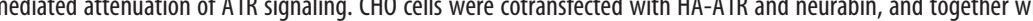
cAMP level is presented as the fold change versus forskolin alone (defined as onefold). ${ }^{*} p<0.05$, R-PIA stimulated versus forskolin A1R signaling. CHO cells were cotransfected with HA-A1R and neurabin, and together with siRNA against RGS4 or control siRNA. Reduction in RGS4 mRNA level was confirmed by RT-PCR. * $p<0.05$, R-PIA stimulated versus forskolin alone; $n=6-8$ for each (n) as onefold); $n=7-8$ for each condition. ${ }^{*} p<0.05$, when compared with the relative cAMP level in neurons of either genotype treated with forskolin alone. \#p $<0.05$, when compared with the relative CAMP level in WT neurons treated with R-PIA and are enhanced in neurabin-deficient mice. The A1R mediates endogenous adenosine-elicited neuroprotection in response to various insults to the brain (Haas and Selbach, 2000; Cunha, 2001; Dunwiddie and Masino, 2001; Stone, 2002). To determine the impact of the A1R-neurabin interaction on the neuroprotective effect of adenosine, we used a kainate-induced seizure model, in which adenosine is released in the brain upon kainate insult and serves as an endogenous anticonvulsant via activation of the A1R (Carswell et al., 1997; Berman et al., 2000). Indeed, we found that blockade of the A1R by the subtype-selective A1R antagonist DPCPX enhanced the severity and progression of kainateinduced seizure activity in WT mice (Fig. 8A), demonstrating a role for the A1R in dampening seizure severity in our model. In contrast, kainate-induced seizures were significantly attenuated in extent and duration in $\mathrm{Nrb}^{-1-}$ mice compared with WT littermates (Fig. $8 \mathrm{~A}, \mathrm{C}$ ), consistent with our observation that sensitivity of endogenous A1R functions is enhanced in $\mathrm{Nrb}^{-/-}$mice (Fig. 4A). While kainate administration at $25 \mathrm{mg} / \mathrm{kg}$ resulted in a 
A

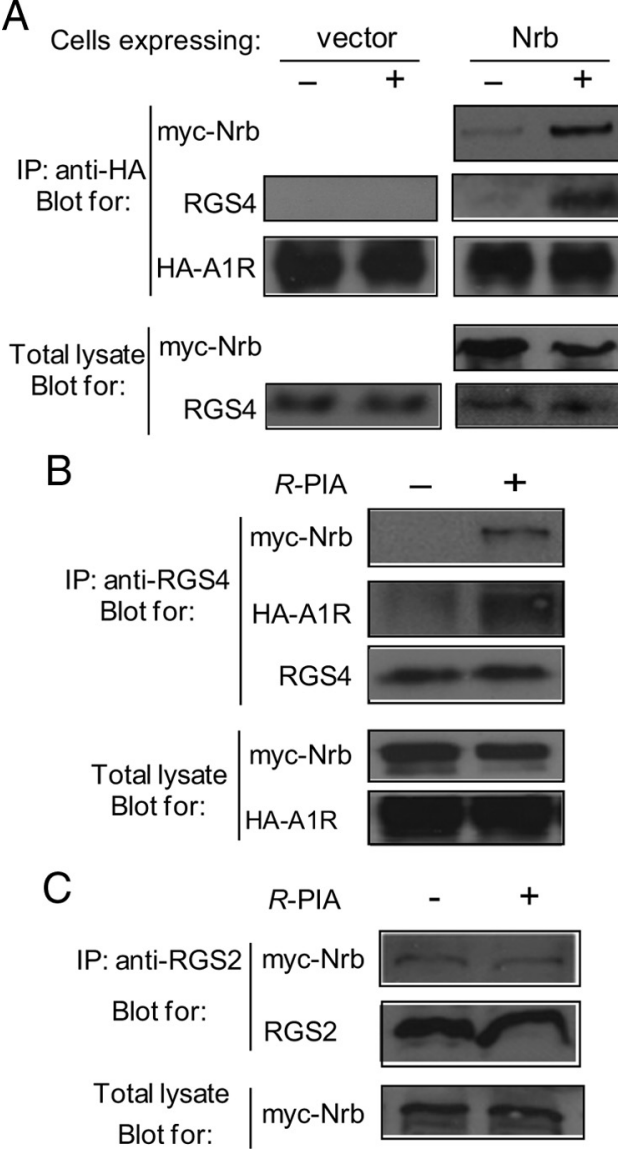

Figure 6. Neurabin (Nrb) scaffolds formation of the A1R-RGS4 complex. A, The A1RRGS4 interaction is dependent upon agonist stimulation and the presence of neurabin. Cells coexpressing HA-A1R and RGS4, together with or without Myc-Nrb, were incubated in the presence or absence of R-PIA for $5 \mathrm{~min}$. Cell lysates were subjected to the immunoisolation assay and analyzed using an anti-HA antibody. Representative blots of at least 3 independent experiments are shown. $\boldsymbol{B}, \mathrm{RGS} 4$ forms a complex with neurabin and the A1R upon agonist stimulation. Cells coexpressing HA-A1R, RGS4, and Myc-Nrb were incubated with or without R-PIA for $5 \mathrm{~min}$. Cell lysates were subjected to the immunoisolation assay using an anti-RGS4 antibody and analyzed by Western blot. Representative blots of multiple independent experiments are shown. C, R-PIA stimulation fails to promote interaction between neurabin and RGS2 in cells. Cells coexpressing A1R, Myc-Nrb, and RGS2 were stimulated with R-PIA for 5 min. Cell lysates were subjected to immunoisolation assay using an anti-RGS2 antibody and analyzed by Western blot. Representative blots of multiple independent experiments are shown. IP, Immunoprecipitation.

nearly $50 \%$ mortality rate in WT mice, all $\mathrm{Nrb}^{-1-}$ mice survived the same insult (Fig. $8 B$ ). Blockade of A1Rs by DPCPX increased the mortality rate to $100 \%$ in both WT and $\mathrm{Nrb}^{-1-}$ mice (Fig. $8 B$ ), further emphasizing the importance of the A1R and its regulation by neurabin in this process.

EEG revealed that $80 \%$ (four of five) of the WT mice that did survive the initial kainate insult developed spontaneous nonconvulsive seizures $\sim 3$ months after injection, whereas only $14 \%$ (one of seven) of $\mathrm{Nrb}^{-1-}$ mice developed spontaneous seizures under the same conditions (Fig. 9A). In addition, the single $\mathrm{Nrb}^{-1-}$ mouse with spontaneous seizure activity exhibited much lower seizure magnitude as measured by EEG compared with WT (Fig. $9 B, C$ ). Importantly, blockade of the A1R during kainate injections by coinjection of the A1R antagonist DPCPX eliminated the observed differences in severity and progression of kainate-induced seizure and death between WT and $\mathrm{Nrb}^{-/-}$ mice (Fig. $8 A, B$ ), confirming that reduced seizure response to kainate in $\mathrm{Nrb}^{-1-}$ mice depends on activation of the A1R. Together, these findings strongly indicate an important role for A1R-neurabin interactions in endogenous adenosine-evoked suppression of seizure progression in vivo, and support the idea that disruption of these interactions, as in our $\mathrm{Nrb}^{-1-}$ mice, enhances the anticonvulsant effect that protects against kainateinduced seizure activity.

Neurons in $\mathrm{Nrb}^{-1-}$ mice are protected from kainateinduced cell death. To further evaluate the role of neurabin in regulating adenosine-evoked neuroprotection at the cellular level, we examined neuronal death in the brains of WT and $\mathrm{Nrb}^{-1-}$ mice that reached similar seizure scores. Severe cell death was observed in the hippocampus of WT mice $7 \mathrm{~d}$ after kainate-induced seizure (Fig. $10 B, E, H$ ). However, in hippocampal slices prepared from $\mathrm{Nrb}^{-1-}$ mice exposed to similar kainate injections, no significant cell death was detected (Fig. 10C, F, I). These morphological findings of neuronal cell survival paralleled seizure and survival findings shown above (Fig. 8), demonstrating that the adenosine-evoked neuroprotective effect against excitotoxic cell death is greatly enhanced when neurabin is not present.

\section{Inhibition of RGS4 enhances the anti-seizure and neuroprotective effects of adenosine in WT but not $\mathrm{Nrb}^{-l-}$ mice}

The in vitro mechanistic studies suggested that neurabin attenuates A1R-mediated signaling by recruiting RGS4 following A1R activation. Therefore, we predicted that inhibition of RGS4 would enhance A1R-evoked neuroprotection in vivo. To test this, we evaluated A1R-mediated suppression of kainate-induced seizures by endogenous adenosine in WT mice with or without coinjection of the RGS4 inhibitor, CCG-4986. Administration of CCG-4986 alone did not cause gross behavioral changes (data not shown), consistent with a previous report that RGS4 knock-out mice lack apparent behavioral defects (Grillet et al., 2005). Significantly, we observed that coinjection of CCG-4986 markedly reduced seizure severity caused by kainate (Fig. 11 A,B). Moreover, coinjection of CCG and kainate resulted in no mortality, compared with a mortality rate of nearly $50 \%$ with kainate insult alone (Fig. 11C). Importantly, coinjection of the A1R antagonist DPCPX eliminated the CCG-4986-dependent attenuation of seizure severity (Fig. $11 \mathrm{~A}-\mathrm{C}$ ), indicating that activation of the A1R is required for the impact of RGS4 inhibition on kainate-evoked seizure activity. These data demonstrate that inhibition of RGS4 enhances endogenous adenosine-evoked anticonvulsant effects through the A1R.

Neurabin expression appears to be required for RGS4 to induce attenuation of A1R signaling (Fig. 5A,D). Therefore, we expected that inhibition of RGS4 would have no effect on A1Rmediated responses in $\mathrm{Nrb}^{-1-}$ mice. Indeed, coinjection of CCG-4986 in Nrb ${ }^{-1-}$ mice did not change seizure behavior induced by kainate in these mutant mice (Fig. 11D). Together with data shown above (Fig. $11 \mathrm{~A}-\mathrm{C}$ ), these results strongly support the interpretation that the neurabin-RGS4 complex is engaged upon agonist activation of the A1R and negatively regulates A1R activity in vivo; disturbance of this complex by one of several complementary strategies enhances the ability of endogenous adenosine to protect against excitotoxin-evoked seizures. In addition, kainate treatment did not significantly affect brain expression levels of either RGS4 or neurabin in WT mice (data not shown), indicating that the A1R/neurabin/RGS4 complex formation is not simply being modulated by altered interacting partner ratios during seizure. 
A

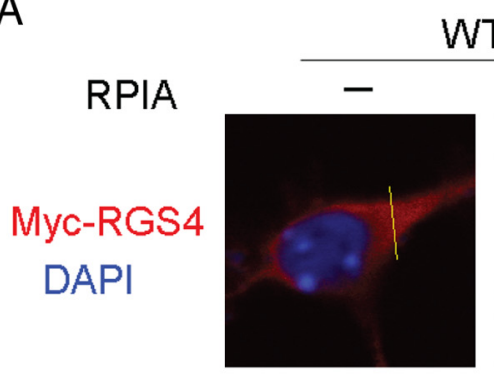

NT
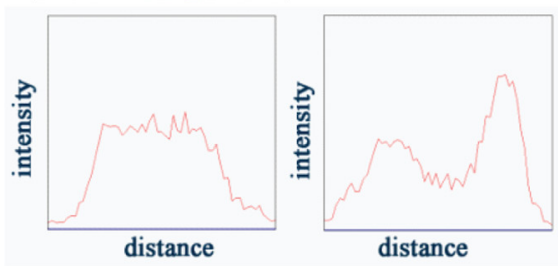

B

solvent

$R$-PIA
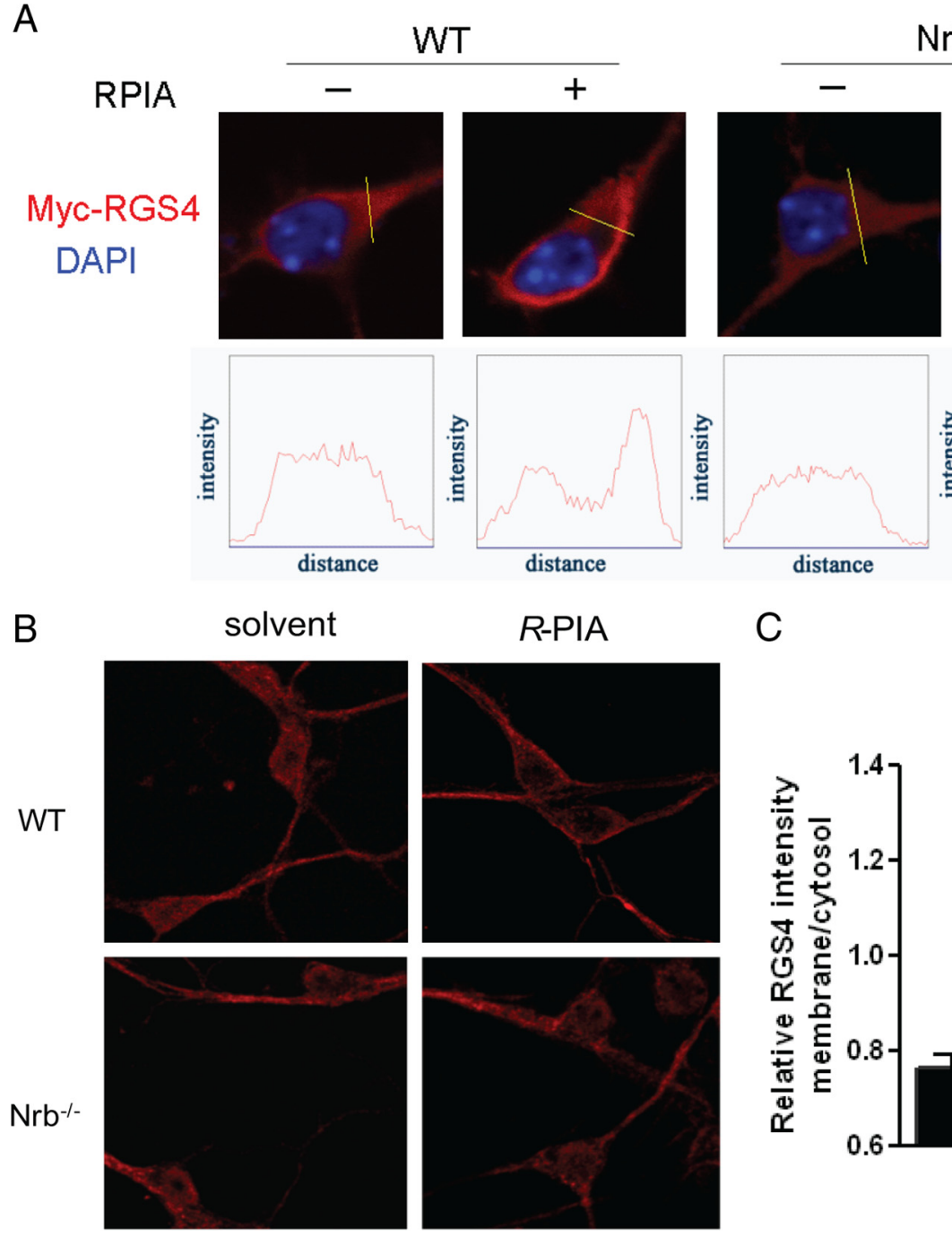

$\mathrm{Nrb}^{-/-}$
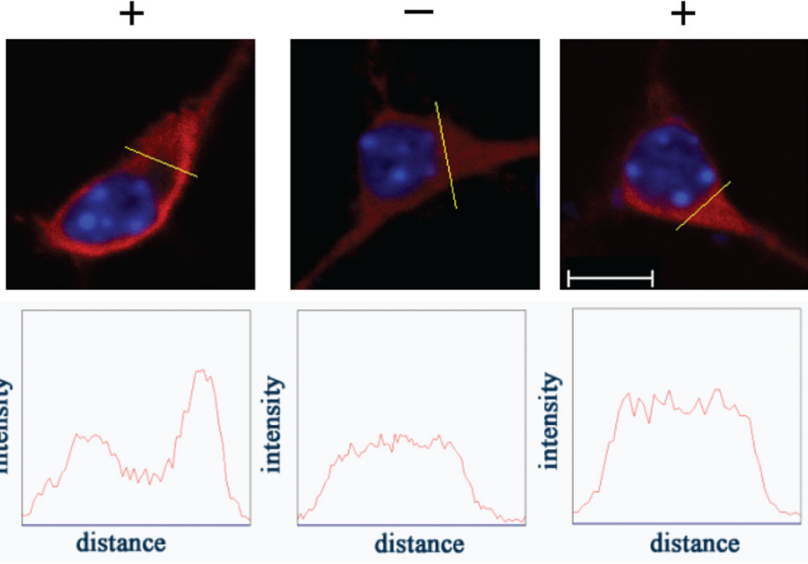

C
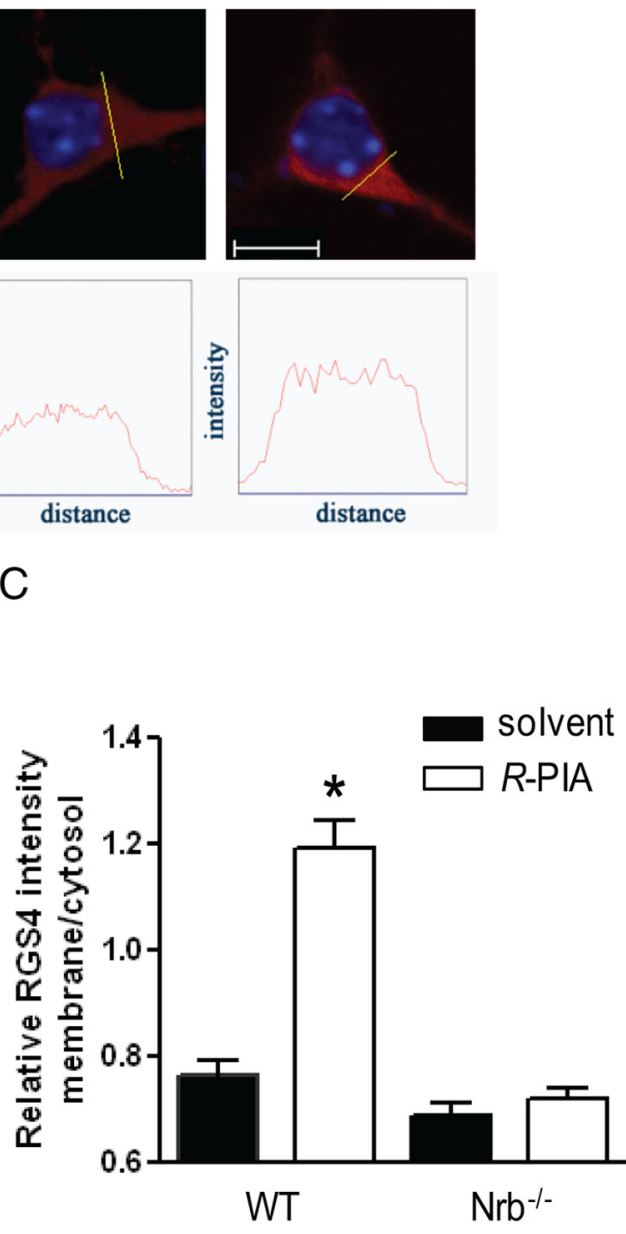

Figure 7. RGS4 is enriched at the plasma membrane upon A1R activation in neurons derived from WT, but not Nrb ${ }^{-1-}$, mice. $A$, Primary cortical neurons cultured from WT or Nrb ${ }^{-/-}$mice were transfected with the Myc-RGS4 plasmid. Representative images of immunostaining with anti-Myc antibody in the presence or absence of R-PIA stimulation. $\boldsymbol{B}$, representative images of distribution of endogenous RGS4 in primary neurons. C, Quantitation of relative RGS4 intensity $(\boldsymbol{B})$ at the plasma membrane over that in the cytoplasm. ${ }^{*} p<0.05$, R-PIA treated versus control. Error bars indicate mean \pm SEM. Thirty to forty neurons from three independent experiments were quantified for each experimental condition.

Kainate-induced neuronal cell death was further evaluated in the brains of WT mice coinjected with the RGS4 inhibitor CCG4986, and compared with that observed in the brains of WT control mice injected with kainate alone. Kainate induced significant cell death in the hippocampus of control mice (Fig. $12 A, C, E)$. However, no obvious cell death was detected in the hippocampus of mice cotreated with CCG-4986 (Fig. 12 B, D,F), demonstrating enhanced neuronal cell survival in these mice. Similarly, kainate failed to cause significant cell death in the hippocampus of $\mathrm{Nrb}^{-1-}$ mice treated with either CCG-4986 or vehicle (data not shown). These data support an enhanced function of endogenous adenosine and the A1R when RGS4 is blocked, which protects neurons from excitotoxic damage.

\section{Discussion}

In the present study, we identify a novel regulation of adenosineevoked neuroprotection by a neural tissue-specific protein, neurabin. As illustrated in Figure 13, neurabin directly interacts with the active A1R upon agonist stimulation and scaffolds complex formation between the A1R and RGS4, which leads to translocation of RGS4 to the plasma membrane and termination of
A1R-induced G-protein signaling. When the neurabin scaffold is absent (as in our $\mathrm{Nrb}^{-1-}$ mice), RGS4 fails to form a complex with the A1R and cannot effectively terminate G-protein signaling. As a result, adenosine-elicited anticonvulsant and neuroprotective effects through the A1R are significantly enhanced. Furthermore, direct inhibition of RGS4 with a small molecule blocker also results in increased G-protein signaling and enhanced neuroprotection in response to adenosine. Together, our study has identified the A1R/neurabin/RGS4 complex as a key regulator in fine-tuning $\mathrm{A} 1 \mathrm{R}$ functions in the nervous system. Disruption of this complex, either by genetic manipulation or by small molecule intervention, enhances A1R-mediated neuroprotection in response to endogenous adenosine without administration of exogenous A1R ligands, which can activate receptors in multiple organs and thereby confound therapeutic specificity.

The anticonvulsant effect of adenosine has long been established and appreciated, and is mainly attributed to the inhibitory function of the A1R (Haas and Selbach, 2000; Cunha, 2001; Dunwiddie and Masino, 2001; Stone, 2002). Indeed, we observe that the severity of kainate-induced seizure is greatly exacerbated in 
A
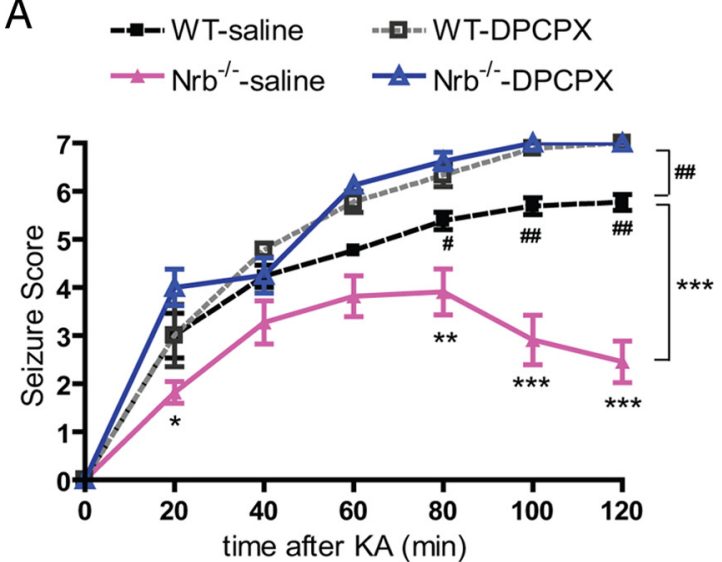

B

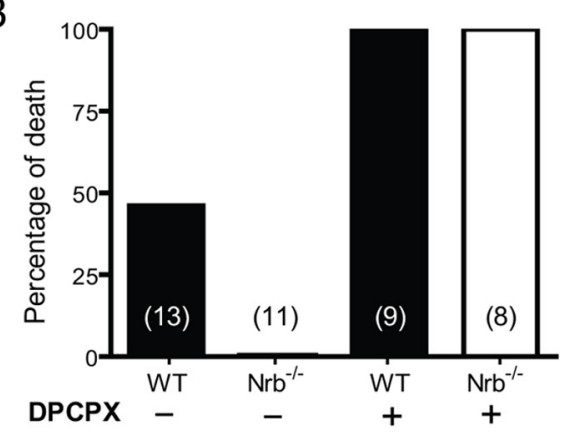

C

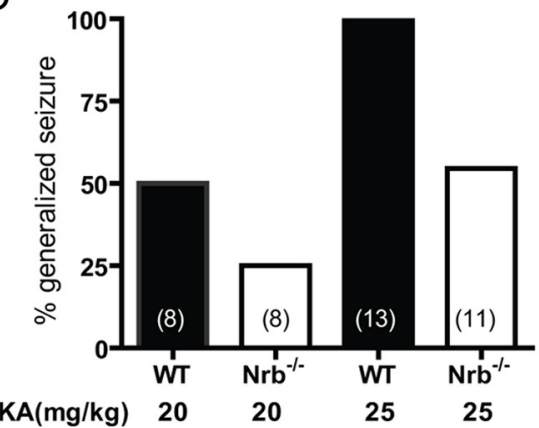

Figure 8. $\mathrm{Nrb}^{-1-}$ mice exhibit enhanced A1R-mediated anticonvulsant effects against kainate-induced seizures. $\boldsymbol{A}$, Seizure severity in response to kainate $(K A)$ is attenuated in $\mathrm{Nrb}^{-1-}$ mice. Seizure activity over time following kainate injection ( $25 \mathrm{mg} / \mathrm{kg}$, i.p.; $n=13$ for WT and $n=11 \mathrm{for} \mathrm{Nrb}^{-1-}$ ) or kainate coinjection with the A1R antagonist DPCPX $(0.5 \mathrm{mg} / \mathrm{kg})$ ( $n=9$ for WT, $n=8$ for $\mathrm{Nrb}^{-1-}$ ) was scored as described in Materials and Methods with a higher score indicating greater seizure severity. ${ }^{*} p<0.05 ;{ }^{* *} p<0.01$; ${ }^{* * *} p<0.001$, $\mathrm{Nrb}^{-1-}$ versus WT mice. ${ }^{\#} p<0.05$; ${ }^{\# \#} p<0.01$, WT mice treated with kainate alone versus kainate plus DPCPX. $\boldsymbol{B}$, Kainate-induced lethality is reduced in $\mathrm{Nrb}^{-1-}$ mice. Data are expressed as a percentage of death in WT $(n=13)$ and $\mathrm{Nrb}^{-1-}(n=11)$ mice caused by administration of kainate $(25 \mathrm{mg} / \mathrm{kg})$ alone or with DPCPX. C, The percentage of mice with generalized seizure is reduced in $\mathrm{Nrb}^{-1-}$ mice. The percentage of mice with generalized seizure within 120 min post-kainate administration at $20 \mathrm{mg} / \mathrm{kg}\left(\mathrm{WT} n=8 ; \mathrm{Nrb}^{-1-} n=8\right)$ or 25 $\mathrm{mg} / \mathrm{kg}\left(\mathrm{WT} n=13 ; \mathrm{Nrb}^{-1-} n=11\right)$ was calculated for each genotype.

mice cotreated with an A1R selective antagonist, DPCPX (Fig. 8). In contrast, when AlR activity is enhanced, as seen in $\mathrm{Nrb}^{-1-}$ mice (Fig. 8) or mice cotreated with the RGS4 inhibitor CCG4986 (Fig. 11), kainate-induced seizure severity is greatly reduced. Significantly, the dose of kainate capable of inducing an $\sim 50 \%$ rate of death in WT mice does not affect $\mathrm{Nrb}^{-1-}$ mice (Fig. 8) or WT mice cotreated with CCG-4986 (Fig. 11). These data demonstrate that enhancement of A1R function by disruption of neurabin and RGS4 provides an effective means to reduce
A

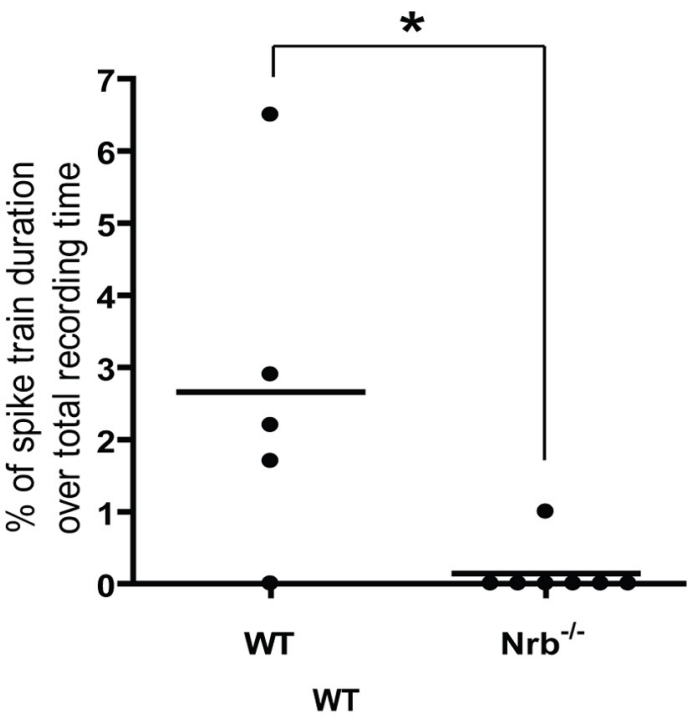

B

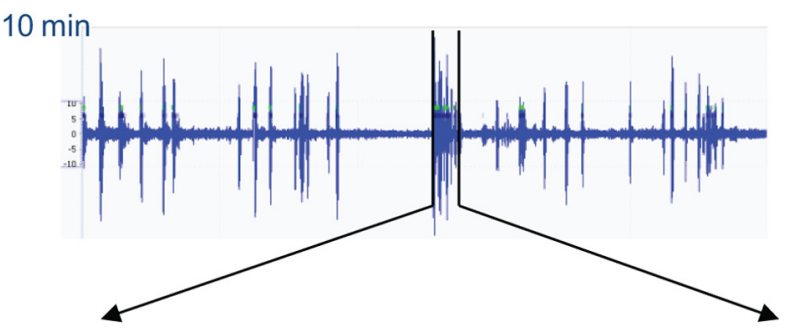

$30 \mathrm{sec}$

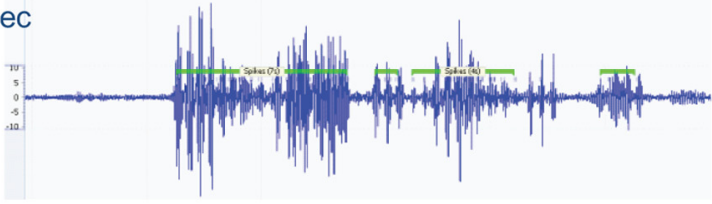

$C_{10 \min } \mathrm{Nrb}^{-/-}$
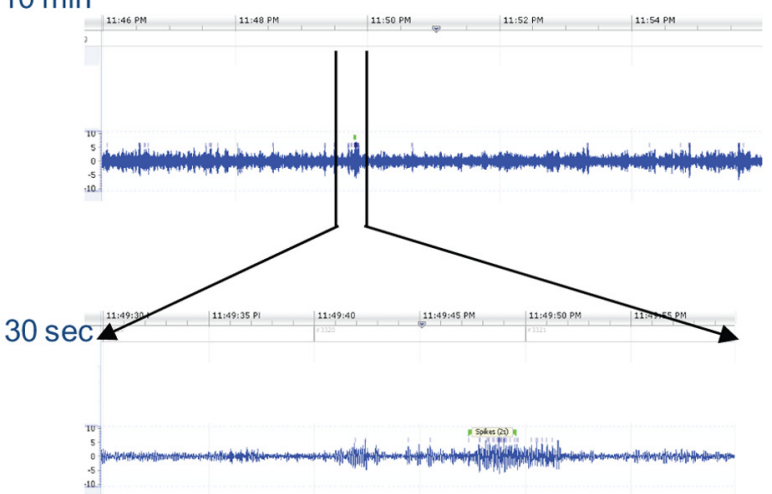

Figure 9. Fewer $\mathrm{Nrb}^{-1-}$ mice than WT mice develop spontaneous seizures $\sim 3$ months after initial kainate insult. $A$, Quantitation of EEG recordings made $\sim 3$ months after the initial kainate injection at $25 \mathrm{mg} / \mathrm{kg}$ in WT and Nrb ${ }^{-1-}$ mice. EEG recordings were performed in mice for $24 \mathrm{~h}$ and the percentage of time in which a spike train was detected (indicating seizure activity in the brain) was calculated for each mouse (represented by single dots). $n=5$ for WT and $n=7$ for Nrb ${ }^{-1-}$ mice. ${ }^{*} p<0.05$. Error bars indicate mean \pm SEM. B, C, Representative EEG recording traces in one WT $(\boldsymbol{B})$ and one $\mathrm{Nrb}^{-1-}(\boldsymbol{C})$ mouse with spontaneous seizures show a much less $\mathrm{EEG}$ magnitude in $\mathrm{Nrb}^{-1-}$ mice than that observed in WT mice with spontaneous seizures. A representative 10 min period with spike train detected is shown for each mouse.

excitotoxic seizures and improve mortality. Given the effectiveness of adenosine in confining seizures in various models, including those for pharmacoresistant seizures (Boison, 2007), several strategies have been proposed to increase extracellular adenosine 
levels to treat epileptic seizures. These strategies include inhibition of adenosine kinase (Boison, 2008), which converts adenosine to AMP; ketogenic diet, which increases global and focal adenosine levels (Masino et al., 2009); and transplantation of adenosine-releasing cells (Boison, 2007). Our present findings open up a new therapeutic direction for seizure control by specifically enhancing A1R activity and responsiveness through disruption of neurabin and RGS4, and provide the first example that a small molecule inhibitor of RGS proteins is effective in reducing excitotoxic seizure through potentiating A1R signaling.

It is well appreciated that basal levels of extracellular adenosine are sufficient to tonically activate adenosine receptors, providing inhibitory purinergic tone in most systems (Dunwiddie and Masino, 2001). Given that neurabin and RGS4 attenuate A1R signaling, we suspect that such tonic activation of A1Rs by adenosine would be enhanced with neurabin deficiency or RGS4 inhibition, leading to stronger inhibition of neuronal excitability and transmission. Therefore, a selective A1R antagonist would be expected to elicit greater potentiation of baseline transmission at synapses in $\mathrm{Nrb}^{-1-}$ mice compared with WT mice or under conditions when RGS4 activity is inhibited. The effects of disrupting the A1R/neurabin/RGS4 complex on synaptic transmission are currently under investigation.

To the best of our knowledge, we have demonstrated for the first time that neurabin directly interacts with a GPCR to negatively regulate receptor activity through an RGS protein. The neurabin homolog spinophilin has been shown to interact with multiple GPCRs, including dopamine D2 receptor (Smith et al., 1999), $\alpha_{2}$ AR (Richman et al., 2001; Wang et al., 2004; Xu et al., 2008), $\alpha_{1}$ AR (Wang et al., 2005), M2 and M3 muscarinic acetylcholine receptors (Kurogi et al., 2009), and the $\mu$-opioid receptor (Charlton et al., 2008); however, direct interaction between neurabin and a GPCR has not been reported. Neurabin has been shown to sequester RGS2 from binding to $\alpha_{1} \mathrm{AR}$, thus enhancing $\alpha_{1}$ AR-mediated signaling (Wang et al., 2007). In contrast, our current study demonstrates that neurabin directly interacts with the active A1R and recruits RGS4 to the A1R complex at the plasma membrane, thereby attenuating A1R-mediated signaling. Meanwhile, the agonist-promoted interaction of the A1R with neurabin seems to have a negligible effect on A1R receptor density (Fig. 4C) and intrinsic affinity for agonist (Fig. 4D) and antagonist binding (data not shown), as these A1R properties are not changed in $\mathrm{Nrb}^{-1-}$ mice.

Direct interaction with a GPCR represents a novel mechanism for neurabin to regulate neuronal activity. Neurabin has been reported to modulate glutamatergic neurotransmission and synaptic plasticity by targeting PP1 to postsynaptic compartments (MacMillan et al., 1999; Terry-Lorenzo et al., 2005; Allen et al., 2006; Hu et al., 2007). Our findings suggest that neurabin attenuates adenosine-evoked inhibition of neuronal activity by interacting with the A1R to diminish its signaling. The sequence of neurabin responsible for interacting with the A1R does not over- lap with the region involved in PP1 binding and regulation (Nakanishi et al., 1997; Burnett et al., 1998) (Figs. 1 B, 2A), making it possible to specifically target neurabin modulation of A1R signaling without altering its function in targeting PP1.

Our findings also provide strong evidence that the ability of neurabin to attenuate endogenous adenosine-elicited neuroprotection is due to the concurrent association of neurabin with both the A1R and RGS4. In vitro assays have demonstrated that neurabin is capable of interacting with several RGS proteins in the R4 subfamily (Wang et al., 2007). However, upon A1R agonist stimulation in intact cells, as shown in Figure 6, neurabin specifically forms a complex with RGS4, but not with its close homolog, RGS2. These data not only demonstrate that the neurabinfacilitated A1R-RGS interaction is highly specific for RGS4, but also suggest that agonist-induced assembly of the A1R/neurabin/ RGS4 complex provides a molecular mechanism by which neurabin expression suppresses A1R responses. Furthermore, the specificity of the interaction of neurabin with RGS4 and not other structurally close family members suggests that neurabin may serve another role in facilitating the selectivity of RGS-mediated attenuation of GPCR signaling.

Our findings that RGS4 inhibitor-dependent reduction of kainate-induced seizure severity is eliminated by cotreatment with an A1R antagonist (Fig. 11) support the hypothesis that A1R signaling is specifically regulated by RGS4 in this seizure model. Moreover, it was recently reported that RGS4 gene deficiency reduced the susceptibility to audiogenic seizures in fragile X mental retardation protein knock-out mice, likely through an increase of $\mathrm{GABA}_{\mathrm{B}}$ receptor signaling (Pacey et al., 2009). Together, these studies suggest that suppression of RGS4 activity may be beneficial for seizure suppression in multiple clinical settings. It is noteworthy that RGS4-null mice are viable and fertile, and lack obvious physiological defects (Grillet et al., 2005). Therefore, pharmacological inhibition of RGS4 is unlikely to cause gross 
A

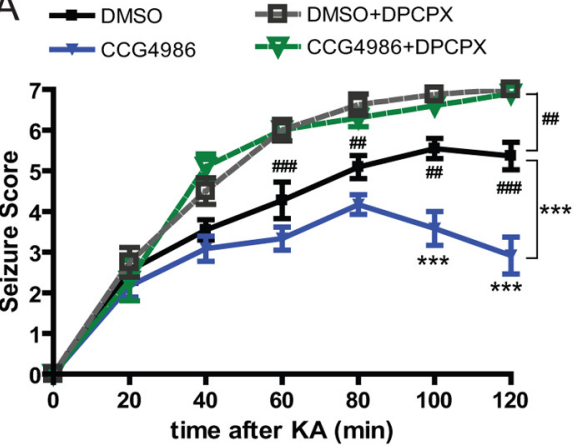

B

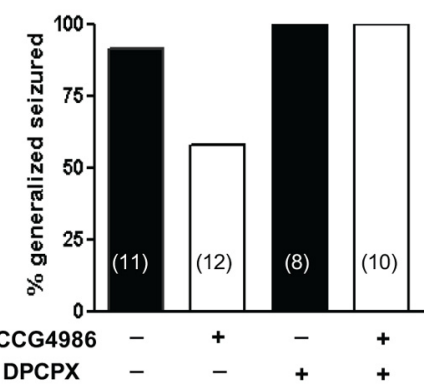

C

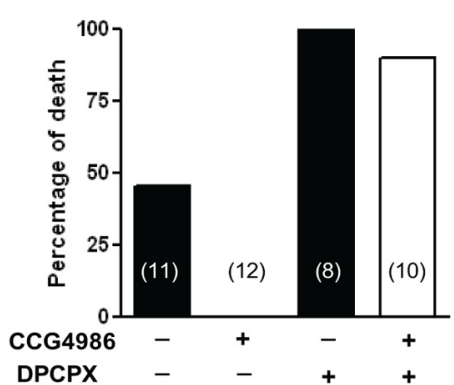

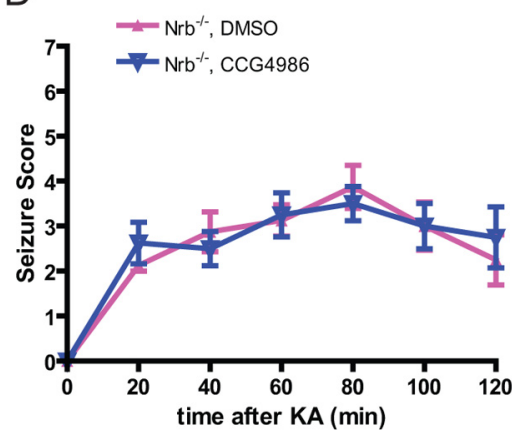

Figure 11. Inhibition of RGS4 enhances the A1R-mediated anti-seizure effect in WT, but not in $\mathrm{Nrb}^{-1-}$ mice. $A$, The RGS4 inhibitor C(G-4986 attenuates kainate (KA)-induced seizure in WT mice. Seizure severity was scored over time postinjection with kainate $(25 \mathrm{mg} / \mathrm{kg})$ in combination with one the following: DMSO vehicle $(n=11) ; C C G-4986(20 \mathrm{mg} / \mathrm{kg})(n=12) ; \mathrm{DPCPX}(0.5$ $\mathrm{mg} / \mathrm{kg})(n=8) ;$; C(G-4986 and DPCPX ( $n=10) .{ }^{* * *} p<0.001$, kainate plus CCG4986 versus kainate alone. ${ }^{\# \#} p<0.01$; ${ }^{\# \#} p<$ 0.001 , kainate alone versus kainate plus DPCPX. $B$, Coinjection of CCG-4986 reduces the percentage of WT mice exhibiting generalized seizure within 120 min post-kainate administration. WT mice treated with kainate alone or in combination with CCG-4986 or DPCPX, or both were compared. C, Coinjection of CCG-4986 reduces kainate-induced lethality in WT mice. Data are expressed as a percentage of death in WT mice treated with kainate alone or in combination with CCG-4986 or DPCPX, or both. $\boldsymbol{D}$, The effect of RGS4 inhibition on kainate-induced seizure is negligible in $\mathrm{Nrb}^{-1-}$ mice. Seizure severity was scored over time postinjection with kainate $(25 \mathrm{mg} / \mathrm{kg})$ in combination with DMSO vehicle $(n=8)$ or CCG-4986 $(20 \mathrm{mg} / \mathrm{kg})(n=8)$. Error bars indicate mean \pm SEM

WT/DMSO
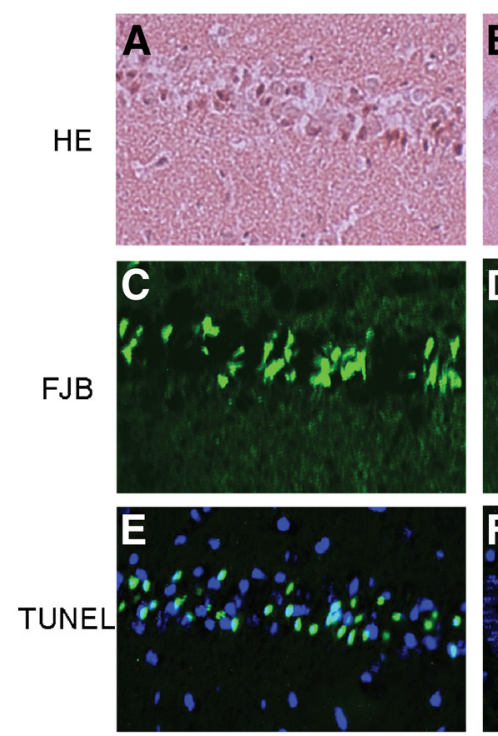

Figure 12. Kainate-induced cell death in the hippocampus is reduced by the RGS4 inhibitor CCG-4986 (CCG). Hippocampal slices of WT mice were collected $7 \mathrm{~d}$ after indicated treatment and stained by hematoxylin and $\operatorname{eosin}(\mathrm{HE}, \boldsymbol{A}, \boldsymbol{B})$. Cell death was detected by Fluoro-Jade B (FJB) staining $(\boldsymbol{C}, \boldsymbol{D})$ and TUNEL assay $(\boldsymbol{E}, \boldsymbol{F})$. Significant neuronal cell death was detected in hippocampus from mice treated with kainate, but not in hippocampus from mice cotreated with kainate (KA) and CCG-4986. abnormality, and hence represents a particularly suitable approach to treat seizures without causing global side effects.

The small molecule CCG-4986 was shown to inhibit both RGS4 interaction with G $\alpha$ o and RGS4 GAP activity in vitro (Roman et al., 2007), likely through modification of multiple cysteine residues including Cys148 at an allosteric site that leads to a conformational change in RGS4 (Roman et al., 2010). Two other RGS proteins that possess a cysteine at equivalent positions, RGS8 and RGS16, cannot be inhibited by CCG-4986 (Roman et al., 2010), suggesting that the allosteric modification by CCG-4986 is unique for RGS4 and that the inhibitory outcome of CCG-4986 is not nonselective in nature. Consistent with our working model that neurabin scaffolds A1R-RGS4 complex formation so that RGS4 can diminish A1R signaling, the effect of CCG-4986 inhibition of RGS4 on A1R-mediated responses requires the presence of neurabin in both cultured cells (Fig. 5A) and in vivo (Fig. $11 D$ ). Our findings regarding CCG-4986 attenuation of kainate-induced seizures provide proof of concept evidence supporting the therapeutic potential of inhibition of RGS4 in neuroprotection.

In summary, we have identified a neural tissue-specific interacting partner of the A1R, neurabin, which attenuates A1R signaling by recruiting RGS4 to form a complex with the receptor. In vivo, endogenous adenosine-evoked protective effects against cell death and kainate-induced seizure are remarkably
enhanced in mice lacking neurabin expression or when RGS4 activity is blocked by a small molecule inhibitor. Significantly, all mice survive the median lethal dose of kainate under these genetic or pharmacological manipulations. Thus, neurabin-RGS4 represents a tissue-selective regulatory mechanism for finetuning adenosine receptor function in neurabin-expressing target cells, i.e., in the nervous system. Disruption of this novel A1R/neurabin/RGS4 complex provides a promising therapeutic strategy for specific enhancement of neuroprotective effects in response to endogenous adenosine released on site on the demand of various insults to the brain, and thus opens a new direction for treatment of pathological conditions that cause neural damage.

\section{References}

Allen JA, Yadav PN, Roth BL (2008) Insights into the regulation of 5-HT2A serotonin receptors by scaffolding proteins and kinases. Neuropharmacology 55:961-968.

Allen PB, Zachariou V, Svenningsson P, Lepore AC, Centonze D, Costa C, Rossi S, Bender G, Chen G, Feng J, Snyder GL, Bernardi G, Nestler EJ, Yan Z, Calabresi P, Greengard P (2006) Distinct roles for spinophilin and neurabin in dopamine-mediated plasticity. Neuroscience 140:897-911.

Bansal G, Druey KM, Xie Z (2007) R4 RGS proteins: regulation of G-protein signaling and beyond. Pharmacol Ther 116:473-495.

Berman DM, Wilkie TM, Gilman AG (1996) GAIP and RGS4 are GTPaseactivating proteins for the $\mathrm{Gi}$ subfamily of $\mathrm{G}$ protein alpha subunits. Cell 86:445-452.

Berman RF, Fredholm BB, Aden U, O'Connor WT (2000) Evidence for 

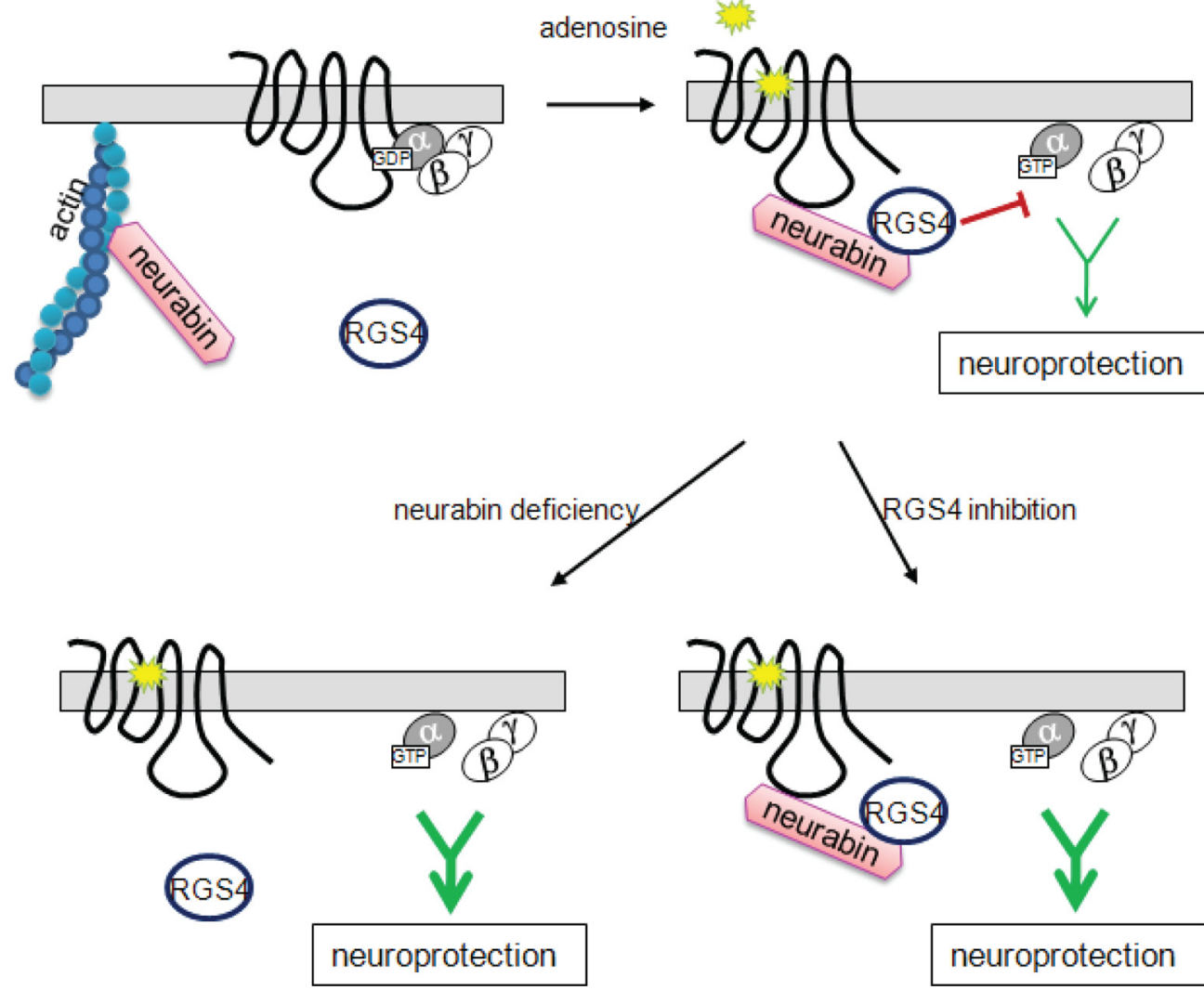

Figure 13. Model of neurabin/RGS4-mediated regulation of A1R signaling. Upon adenosine binding, neurabin directly interacts with both the active A1R and RGS4 and scaffolds formation of the A1R/neurabin/RGS4 complex, which attenuates A1R-induced G-protein signaling and neuroprotective effects. However, when the neurabin scaffold is absent (as in our Nrb ${ }^{-1-}$ mice), RGS4 cannot translocate to the plasma membrane or form a complex with the A1R, thus rendering it unable to effectively terminate G-protein signaling. Also, A1R-induced G-protein signaling is enhanced when RGS4 activity is inhibited (as by the small molecule blocker). In both cases, adenosine-elicited anticonvulsant and neuroprotective effects mediated through the A1R are greatly improved.

increased dorsal hippocampal adenosine release and metabolism during pharmacologically induced seizures in rats. Brain Res 872:44-53.

Bockaert J, Perroy J, Bécamel C, Marin P, Fagni L (2010) GPCR interacting proteins (GIPs) in the nervous system: roles in physiology and pathologies. Annu Rev Pharmacol Toxicol 50:89-109.

Boison D (2007) Adenosine-based cell therapy approaches for pharmacoresistant epilepsies. Neurodegener Dis 4:28-33.

Boison D (2008) The adenosine kinase hypothesis of epileptogenesis. Prog Neurobiol 84:249-262.

Brady AE, Wang Q, Colbran RJ, Allen PB, Greengard P, Limbird LE (2003) Spinophilin stabilizes cell surface expression of alpha 2B-adrenergic receptors. J Biol Chem 278:32405-32412.

Burnett PE, Blackshaw S, Lai MM, Qureshi IA, Burnett AF, Sabatini DM, Snyder SH (1998) Neurabin is a synaptic protein linking p70 S6 kinase and the neuronal cytoskeleton. Proc Natl Acad Sci U S A 95:8351-8356.

Carswell HV, Graham DI, Stone TW (1997) Kainate-evoked release of adenosine from the hippocampus of the anaesthetised rat: possible involvement of free radicals. J Neurochem 68:240-247.

Charlton JJ, Allen PB, Psifogeorgou K, Chakravarty S, Gomes I, Neve RL, Devi LA, Greengard P, Nestler EJ, Zachariou V (2008) Multiple actions of spinophilin regulate mu opioid receptor function. Neuron 58:238-247.

Chatterjee TK, Fisher RA (2000) Cytoplasmic, nuclear, and Golgi localization of RGS proteins. Evidence for N-terminal and RGS domain sequences as intracellular targeting motifs. J Biol Chem 275:24013-24021.

Cottingham C, Chen Y, Jiao K, Wang Q (2011) The antidepressant desipramine is an arrestin-biased ligand at the $\alpha 2 \mathrm{~A}$-adrenergic receptor driving receptor down-regulation in vitro and in vivo. J Biol Chem 286:36063-36075.

Cunha RA (2001) Adenosine as a neuromodulator and as a homeostatic regulator in the nervous system: different roles, different sources and different receptors. Neurochem Int 38:107-125.

Dunwiddie TV, Masino SA (2001) The role and regulation of adenosine in the central nervous system. Annu Rev Neurosci 24:31-55.

Ferguson SS (2001) Evolving concepts in G protein-coupled receptor endo- cytosis: the role in receptor desensitization and signaling. Pharmacol Rev 53:1-24.

Fredholm BB, Chen JF, Masino SA, Vaugeois JM (2005) Actions of adenosine at its receptors in the CNS: insights from knockouts and drugs. Annu Rev Pharmacol Toxicol 45:385-412.

Grillet N, Pattyn A, Contet C, Kieffer BL, Goridis C, Brunet JF (2005) Generation and characterization of Rgs4 mutant mice. Mol Cell Biol 25:4221-4228.

Haas HL, Selbach O (2000) Functions of neuronal adenosine receptors. Naunyn Schmiedebergs Arch Pharmacol 362:375-381.

Hanyaloglu AC, von Zastrow M (2008) Regulation of GPCRs by endocytic membrane trafficking and its potential implications. Annu Rev Pharmacol Toxicol 48:537-568.

Hollinger S, Hepler JR (2002) Cellular regulation of RGS proteins: modulators and integrators of G protein signaling. Pharmacol Rev 54:527-559.

Huang C, Hepler JR, Gilman AG, Mumby SM (1997) Attenuation of Giand $\mathrm{Gq}$-mediated signaling by expression of RGS4 or GAIP in mammalian cells. Proc Natl Acad Sci U S A 94:6159-6163.

Hu XD, Huang Q, Yang X, Xia H (2007) Differential regulation of AMPA receptor trafficking by neurabin-targeted synaptic protein phosphatase-1 in synaptic transmission and long-term depression in hippocampus. J Neurosci 27:4674-4686.

Kurogi M, Nagatomo K, Kubo Y, Saitoh O (2009) Effects of spinophilin on the function of RGS8 regulating signals from M2 and M3-mAChRs. Neuroreport 20:1134-1139.

Lefkowitz RJ (2007) Seven transmembrane receptors: something old, something new. Acta Physiol (Oxf) 190:9-19.

MacMillan LB, Bass MA, Cheng N, Howard EF, Tamura M, Strack S, Wadzinski BE, Colbran RJ (1999) Brain actin-associated protein phosphatase 1 holoenzymes containing spinophilin, neurabin, and selected catalytic subunit isoforms. J Biol Chem 274:35845-35854.

Maemoto T, Finlayson K, Olverman HJ, Akahane A, Horton RW, Butcher SP (1997) Species differences in brain adenosine A1 receptor pharmacology 
revealed by use of xanthine and pyrazolopyridine based antagonists. $\mathrm{Br} \mathrm{J}$ Pharmacol 122:1202-1208.

Masino SA, Kawamura M, Wasser CD, Wasser CA, Pomeroy LT, Ruskin DN (2009) Adenosine, ketogenic diet and epilepsy: the emerging therapeutic relationship between metabolism and brain activity. Curr Neuropharmacol 7:257-268.

Muly EC, Allen P, Mazloom M, Aranbayeva Z, Greenfield AT, Greengard P (2004) Subcellular distribution of neurabin immunolabeling in primate prefrontal cortex: comparison with spinophilin. Cereb Cortex 14:13981407.

Murphy MG, Moak CM, Byczko Z, MacDonald WF (1991) Adenosinedependent regulation of cyclic AMP accumulation in primary cultures of rat astrocytes and neurons. J Neurosci Res 30:631-640.

Nakanishi H, Obaishi H, Satoh A, Wada M, Mandai K, Satoh K, Nishioka H, Matsuura Y, Mizoguchi A, Takai Y (1997) Neurabin: a novel neural tissue-specific actin filament-binding protein involved in neurite formation. J Cell Biol 139:951-961.

Neitzel KL, Hepler JR (2006) Cellular mechanisms that determine selective RGS protein regulation of $\mathrm{G}$ protein-coupled receptor signaling. Semin Cell Dev Biol 17:383-389.

Neubig RR, Siderovski DP (2002) Regulators of G-protein signalling as new central nervous system drug targets. Nat Rev Drug Discov 1:187-197.

Oliver CJ, Terry-Lorenzo RT, Elliott E, Bloomer WA, Li S, Brautigan DL, Colbran RJ, Shenolikar S (2002) Targeting protein phosphatase 1 (PP1) to the actin cytoskeleton: the neurabin I/PP1 complex regulates cell morphology. Mol Cell Biol 22:4690-4701.

Pacey LK, Heximer SP, Hampson DR (2009) Increased GABA(B) receptormediated signaling reduces the susceptibility of fragile $\mathrm{X}$ knockout mice to audiogenic seizures. Mol Pharmacol 76:18-24.

Reiter E, Lefkowitz RJ (2006) GRKs and beta-arrestins: roles in receptor silencing, trafficking and signaling. Trends Endocrinol Metab 17:159165.

Reppert SM, Weaver DR, Stehle JH, Rivkees SA (1991) Molecular cloning and characterization of a rat A1-adenosine receptor that is widely expressed in brain and spinal cord. Mol Endocrinol 5:1037-1048.

Richman JG, Brady AE, Wang Q, Hensel JL, Colbran RJ, Limbird LE (2001) Agonist-regulated interaction between alpha 2-adrenergic receptors and spinophilin. J Biol Chem 276:15003-15008.

Ritter SL, Hall RA (2009) Fine-tuning of GPCR activity by receptorinteracting proteins. Nat Rev Mol Cell Biol 10:819-830.

Roman DL, Talbot JN, Roof RA, Sunahara RK, Traynor JR, Neubig RR (2007) Identification of small-molecule inhibitors of RGS4 using a highthroughput flow cytometry protein interaction assay. Mol Pharmacol 71:169-175.

Roman DL, Blazer LL, Monroy CA, Neubig RR (2010) Allosteric inhibition of the regulator of $\mathrm{G}$ protein signaling-Galpha protein-protein interaction by CCG-4986. Mol Pharmacol 78:360-365.

Ross EM, Wilkie TM (2000) GTPase-activating proteins for heterotrimeric G proteins: regulators of $\mathrm{G}$ protein signaling (RGS) and RGS-like proteins. Annu Rev Biochem 69:795-827.
Schauwecker PE, Steward O (1997) Genetic determinants of susceptibility to excitotoxic cell death: implications for gene targeting approaches. Proc Natl Acad Sci U S A 94:4103-4108.

Scholl DJ, Wells JN (2000) Serine and alanine mutagenesis of the nine native cysteine residues of the human A(1) adenosine receptor. Biochem Pharmacol 60:1647-1654.

Sebastião AM, Ribeiro JA (2009) Adenosine receptors and the central nervous system. Handb Exp Pharmacol 193:471-534.

Sjögren B, Neubig RR (2010) Thinking outside of the "(RGS) box": new approaches to therapeutic targeting of regulators of $\mathrm{G}$ protein signaling. Mol Pharmacol 78:550-557.

Smith FD, Oxford GS, Milgram SL (1999) Association of the D2 dopamine receptor third cytoplasmic loop with spinophilin, a protein phosphatase1-interacting protein. J Biol Chem 274:19894-19900.

Srinivasa SP, Bernstein LS, Blumer KJ, Linder ME (1998) Plasma membrane localization is required for RGS4 function in Saccharomyces cerevisiae. Proc Natl Acad Sci U S A 95:5584-5589.

Stone TW (2002) Purines and neuroprotection. Adv Exp Med Biol 513:249-280

Terry-Lorenzo RT, Roadcap DW, Otsuka T, Blanpied TA, Zamorano PL, Garner CC, Shenolikar S, Ehlers MD (2005) Neurabin/protein phosphatase- 1 complex regulates dendritic spine morphogenesis and maturation. Mol Biol Cell 16:2349-2362.

Wang Q, Limbird LE (2002) Regulated interactions of the alpha 2A adrenergic receptor with spinophilin, 14-3-3zeta, and arrestin 3. J Biol Chem 277:50589-50596.

Wang Q, Zhao J, Brady AE, Feng J, Allen PB, Lefkowitz RJ, Greengard P, Limbird LE (2004) Spinophilin blocks arrestin actions in vitro and in vivo at G protein-coupled receptors. Science 304:1940-1944.

Wang X, Zeng W, Soyombo AA, Tang W, Ross EM, Barnes AP, Milgram SL, Penninger JM, Allen PB, Greengard P, Muallem S (2005) Spinophilin regulates $\mathrm{Ca} 2+$ signalling by binding the $\mathrm{N}$-terminal domain of RGS2 and the third intracellular loop of G-protein-coupled receptors. Nat Cell Biol 7:405-411.

Wang X, Zeng W, Kim MS, Allen PB, Greengard P, Muallem S (2007) Spinophilin/neurabin reciprocally regulate signaling intensity by $\mathrm{G}$ proteincoupled receptors. EMBO J 26:2768-2776.

Witt-Enderby PA, Jarzynka MJ, Krawitt BJ, Melan MA (2004) Knock-down of RGS4 and beta tubulin in CHO cells expressing the human MT1 melatonin receptor prevents melatonin-induced receptor desensitization. Life Sci 75:2703-2715.

Xie GX, Palmer PP (2007) How regulators of G protein signaling achieve selective regulation. J Mol Biol 366:349-365.

Xu J, Chen Y, Lu R, Cottingham C, Jiao K, Wang Q (2008) Protein kinase A phosphorylation of spinophilin modulates its interaction with the $\alpha 2 \mathrm{~A}$ adrenergic receptor (AR) and alters temporal properties of $\alpha 2 \mathrm{AAR}$ internalization. J Biol Chem 283:14516-14523.

Yang DD, Kuan CY, Whitmarsh AJ, Rincón M, Zheng TS, Davis RJ, Rakic P, Flavell RA (1997) Absence of excitotoxicity-induced apoptosis in the hippocampus of mice lacking the Jnk3 gene. Nature 389:865-870. 